\title{
mblmpute: an accurate and robust imputation method for microbiome data
}

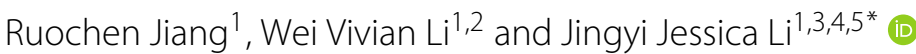

\author{
*Correspondence: jli@stat.ucla.edu \\ 1 Department of Statistics, University \\ of California, Los Angeles \\ 90095-1554, CA, USA \\ ${ }^{3}$ Department of Human Genetics, \\ University of California, Los Angeles \\ 90095-7088, CA, USA \\ Full list of author information is \\ available at the end of the article
}

\begin{abstract}
A critical challenge in microbiome data analysis is the existence of many non-biological zeros, which distort taxon abundance distributions, complicate data analysis, and jeopardize the reliability of scientific discoveries. To address this issue, we propose the first imputation method for microbiome data-mblmpute-to identify and recover likely non-biological zeros by borrowing information jointly from similar samples, similar taxa, and optional metadata including sample covariates and taxon phylogeny. We demonstrate that mblmpute improves the power of identifying disease-related taxa from microbiome data of type 2 diabetes and colorectal cancer, and mblmpute preserves non-zero distributions of taxa abundances.
\end{abstract}

\section{Introduction}

Microbiome studies explore the collective genomes of microorganisms living in a certain environment such as soil, sea water, animal skin, and human gut. Numerous studies have confirmed the importance of microbiomes in natural environments and human bodies [1]. For example, new discoveries have revealed the important roles microbiomes play in complex diseases such as obesity [2], diabetes [3], pulmonary disease [4,5], and cancers [6]. These studies have shown the potential of human microbes as biomarkers for disease diagnosis or as therapeutic targets for disease treatment [7].

The development of high-throughput sequencing technologies has advanced microbiome studies in the last decade [8]. Two sequencing technologies are primarily used: the 16S ribosomal RNA (rRNA) amplicon sequencing and the shotgun metagenomic sequencing. The $16 \mathrm{~S}$ rRNA amplicon sequencing measures $16 \mathrm{~S}$ rRNAs, which can be used to identify and distinguish microbes [9]. The 16S rRNA sequencing reads are either clustered into operational taxonomic units (OTUs) [10] or mapped to amplicon sequence variants (ASVs) $[11,12]$. The shotgun metagenomic sequencing, also known as the wholegenome sequencing (WGS), sequences all DNAs in a microbiome sample, including whole genomes of microbial species and host DNAs [10, 13-19]. The WGS sequencing reads are mapped to known microbial genome databases to quantify the abundances of

(c) The Author(s). 2021 Open Access This article is licensed under a Creative Commons Attribution 4.0 International License which permits use, sharing, adaptation, distribution and reproduction in any medium or format, as long as you give appropriate credit to the original author(s) and the source, provide a link to the Creative Commons licence, and indicate if changes were made. The images or other third party material in this article are included in the article's Creative Commons licence, unless indicated otherwise in a credit line to the material. If material is not included in the article's Creative Commons licence and your intended use is not permitted by statutory regulation or exceeds the permitted use, you will need to obtain permission directly from the copyright holder. To view a copy of this licence, visit http://creativecommons.org/licenses/by/4.0/. The Creative Commons Public Domain Dedication waiver (http://creativecommons.org/publicdomain/zero/1.0/) applies to the data made available in this article, unless otherwise stated in a credit line to the data. 
microbial species. Despite the vast differences between the two technologies, $16 \mathrm{~S}$ and WGS data can both be processed into the same data structure containing abundances of microbes in microbiome samples: a taxon count matrix with rows as microbiome samples (which often correspond to subjects or individuals) and columns as taxa (i.e., OTUs or ASVs for 16S rRNA data and species for WGS data), and each entry corresponds to the number of reads mapped to a taxon in a microbiome sample. It is worth noting that the total read count per microbiome sample, i.e., the sum of entries in a row of the count matrix, differs by five orders of magnitude between the two technologies: $\sim 10^{3}$ per sample for $16 \mathrm{~S}$ rRNA data and $\sim 10^{8}$ for WGS data [20].

A critical challenge in microbiome data analysis is the existence of many zeros in taxon counts, an ubiquitous phenomenon for both 16S rRNA and WGS data [20]. The large proportion of zeros belongs to three categories by origin: biological, technical, and sampling zeros [21]. Biological zeros represent true zero abundances of non-existent taxa in microbiome samples. In contrast, technical and sampling zeros are non-biological zeros with different origins: technical zeros arise from pre-sequencing experimental artifacts (e.g., DNA degradation during library preparation and inefficient sequence amplification due to factors such as GC content bias) [22], while sampling zeros are due to limited sequencing depths. Although WGS data have much larger per-sample total read counts than $16 \mathrm{~S}$ data have, they still suffer from sampling zeros because they sequence more nucleic acid sequences (microbial genomes instead of $16 \mathrm{~S}$ rRNAs) and their effective sequencing depths are reduced by widespread host DNA contaminations [23-25].

This data sparsity issue challenges microbiome data analysis, as most state-of-the-art methods have poor performance on data containing too many zeros. Adding a pseudocount of one to zeros is a common, simple approach [26, 27], but it is ad hoc and suboptimal because it cannot distinguish biological zeros from technical and sampling zeros [28, 29]. Kaul et al. [30] developed an approach to distinguish these three types of zeros and to correct only the sampling zeros; however, their correction is still a simple addition of a pseudo-count of one, ignoring the fact that the (unobserved) actual counts of sampling zeros may not be exactly one.

In particular, this data sparsity issue hinders the differentially abundant (DA) taxon analysis, which aims to identify the taxa that exhibit significantly different abundances between two groups of samples [13]. Microbiome researchers employ two major types of statistical methods to identify DA taxa. Methods of the first type use parametric models [7, 26, 31-38]. For example, the zero-inflated negative binomial generalized linear model (ZINB-GLM) is used in [7, 31, 32], the negative binomial regression is used in the DESeq2-phyloseq method [33,34], and the zero-inflated Gaussian model is used in the metagenomeSeq method [35]. However, these parametric model assumptions may not hold for a particular dataset [39]. Methods of the second type perform non-parametric statistical tests that do not assume specific data distributions. Widely used methods include the Wilcoxon rank-sum test [14-19] and ANCOM [27]. A major drawback of these non-parametric methods is that a taxon would be called DA if its zero proportions differ significantly between two groups of samples, but this difference is unlikely biologically meaningful due to the prevalence of technical and sampling zeros. Note that both types of DA methods require the input taxon abundances to be in one of three units: counts [7, 31, 32, 34], log-transformed counts [35], and proportions (i.e., each taxon's count is divided by the sum of all taxa's counts in a sample) [26, 27, 36-38]; regardless of 
the unit, DA taxon analysis is always biased by the prevalence of technical and sampling zeros.

In addition to DA taxon analysis, other microbiome data analyses, such as the construction of taxon interaction networks [40-43], are also impeded by the data sparsity challenge. Although zero-inflated modeling is commonly used for sparse data, it requires a specific model formulation for each analysis task, which is often complicated or unrealistic for most microbiome researchers. Hence, a flexible and robust approach is needed to address the sparsity issue of microbiome data.

Imputation is a widely used technique to recover missing data and facilitate data analysis. It has successful applications in many fields, e.g., recommender systems (e.g., the Netflix challenge [44]), image and speech reconstruction [45-47], imputation of unmeasured epigenomics datasets [48], missing genotype prediction in genome-wide association studies [49], and the more recent gene expression recovery in single-cell RNAsequencing (scRNA-seq) data [50-54]. Microbiome and scRNA-seq data have the same count matrix structure if one considers microbiome samples and taxa as analogs to cells and genes, respectively; both data have large proportions of non-biological zeros. Given the successes of scRNA-seq imputation methods, we hypothesize that imputation can also relieve the data sparsity issue in microbiome data. Although there are methods utilizing matrix completion in the microbiome field, their main purpose is to perform community detection or dimension reduction instead of imputation $[55,56]$. Two distinct features of microbiome data make it suboptimal to directly apply existing imputation methods. First, microbiome data are often accompanied by metadata including sample covariates and taxon phylogeny, which, however, cannot be used by existing imputation methods. In particular, phylogenetic information is known to be valuable for microbiome data analysis [57-64], as closely-related taxa in a phylogeny are likely to have similar functions and abundances in samples [65-68]. Second, microbiome data have a much smaller number of samples (often in hundreds) than the number of cells (often in tens of thousands) in scRNA-seq data, making those deep-learning based imputation methods inapplicable $[54,69]$. On the other hand, the smaller sample size allows microbiome data to afford an imputation method that focuses more on imputation accuracy than computational time.

Here, we propose mbImpute, the first imputation method designed for microbiome data including both $16 \mathrm{~S}$ and WGS data. The mbImpute method identifies and corrects the zeros and low counts that are unlikely biological (for ease of terminology, we will refer to them as non-biological zeros in the following text) in microbiome taxon count data. The goal of mbImpute is to provide a principled data-driven approach to relieve the microbiome data sparsity issue due to prevalent non-biological zeros. To achieve this, mbImpute leverages three sources of information: a taxon count matrix, sample covariates (e.g., sample library size and subjects' age, gender, and body mass index), and taxon phylogeny, with the latter two sources being optional. There are two main steps in mbImpute (Fig. 1): first, mbImpute identifies likely non-biological zeros; second, it imputes these zeros by borrowing information from similar taxa (determined by both phylogeny and counts), similar microbiome samples (in terms of taxon counts), and sample covariates if available (see an illustration of the imputation step in Additional file 1: Figure S1). The imputed data are expected to contain recovered taxon counts and would thus facilitate various downstream analyses, such as the identification of DA taxa and the construction of taxon interaction networks. Microbiome researchers can use mbImpute 


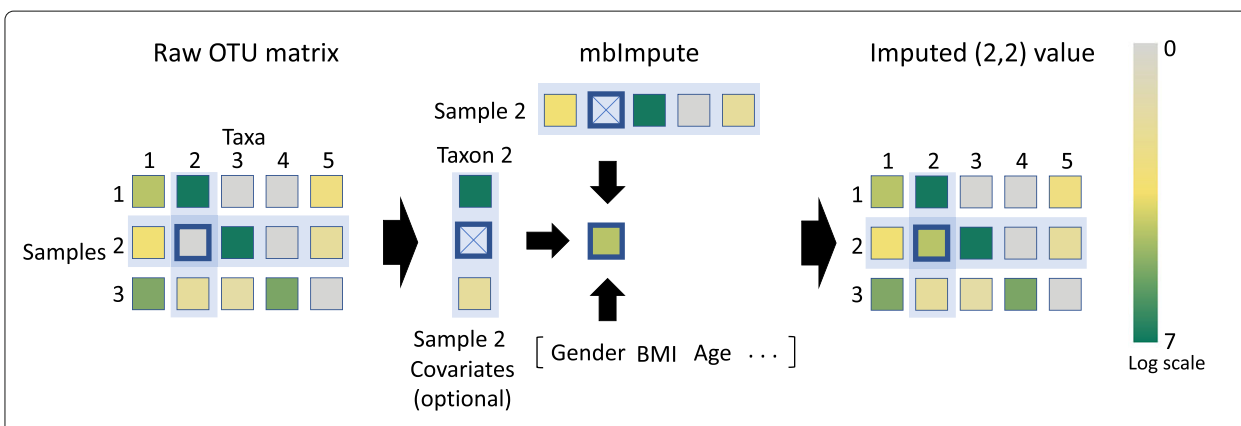

Fig. 1 An illustration of mblmpute. After mblmpute identifies likely non-biological zeros, it imputes them (e.g., the abundance of taxon 2 in sample 2) by jointly borrowing information from similar samples, similar taxa, and sample covariates if available (details in Methods)

to avoid the hassle of dealing with sparse data in individual analysis tasks and to enjoy the flexibility of building up data analysis pipelines.

\section{Results}

mblmpute outperforms non-microbiome imputation methods in recovering missing taxon abundances and empowering DA taxon identification

As there are no imputation methods for microbiome data, we benchmark mbImpute against five state-of-the-art imputation methods designed for non-microbiome data: four popular scRNA-seq imputation methods (scImpute [50], SAVER [52], MAGIC [51], and ALRA [53]) and a widely used general imputation method softImpute [70]. We design two simulation studies, and the common goal is to obtain a "complete" microbiome dataset without non-biological zeros, so that we can evaluate imputation accuracy by comparing the imputed data with the complete data. In the first study, we simulate complete data from a generative model fitted to a WGS dataset of type 2 diabetes (T2D) samples [18]; In the second, more realistic simulation study, we extract a sub-dataset with fewer than $15 \%$ zeros as the complete data from another WGS dataset of T2D samples [19]. In both simulation studies (see Additional file 1: Simulation 1 and Simulation $2[1-3,6-8,10,13-$ $19,26,27,30-32,50,52,54,70-101])$, we introduce non-biological zeros into the complete data by mimicking the observed zero patterns in real datasets, obtaining what we call the zero-inflated data. After applying the six imputation methods to the zero-inflated data in both studies, we compare these methods' imputation accuracy in three aspects: (1) the mean squared error (MSE) between the imputed data and the complete data, (2) each taxon's Pearson correlation between its imputed abundances and complete abundances, and (3) the Wasserstein distance between the distributions of taxa's abundance mean/(standard deviation) ratios in the imputed data and the complete data. Figure $2 \mathrm{a}-$ $\mathrm{d}$ illustrate the comparison results, indicating that mbImpute achieves the best overall performance in all three aspects. In particular, Fig. 2c-d and Additional file 1: Figure S2 show that the imputed data by mbImpute best resemble the complete data, verifying the advantage of mbImpute in recovering missing taxon abundances in microbiome data.

We next demonstrate that mbImpute is a robust method. The core of mbImpute is to borrow three-way information from similar samples, similar taxa, and sample covariates to impute non-biological zeros in microbiome data (see Methods). In the aforementioned second simulation study (Additional file 1: Simulation 2), we scramble samples in the real T2D WGS data when we select the complete data, a situation not optimal for mbImpute; 


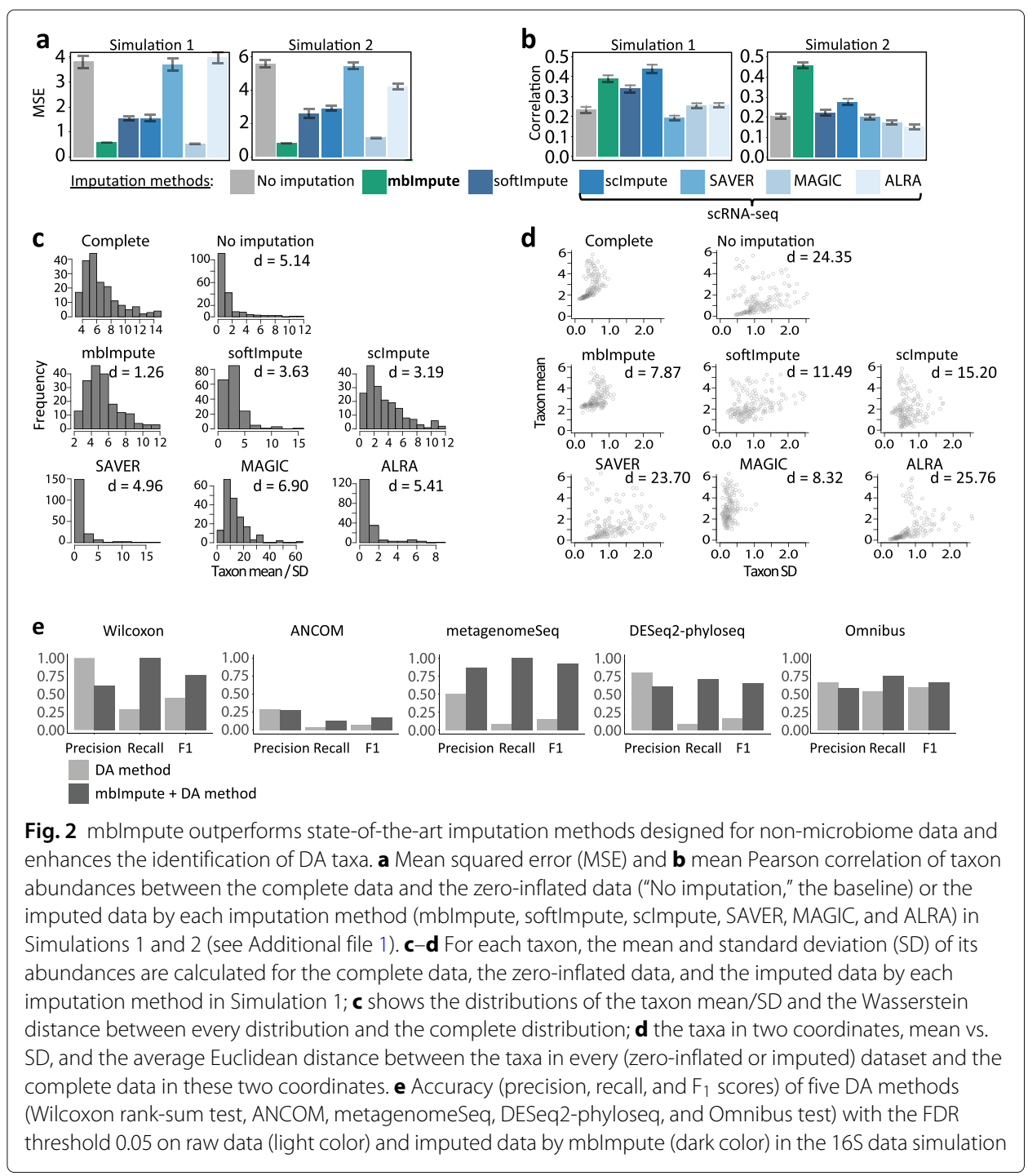

however, mbImpute still outperforms existing imputation methods (Fig. 2a, b). To further test for the robustness of mbImpute, we design a third simulation study including four simulation schemes, where the information useful for imputation is encoded in sample covariates only, samples only, taxa only, or three sources together (see Additional file 1: Simulation 3). Additional file 1: Figure S3 shows that mbImpute effectively recovers non-biological zeros and reduces the MSE under every scheme. These results verify the robustness of mbImpute in selectively leveraging the information useful for imputation.

To further evaluate the performance of mbImpute on $16 \mathrm{~S}$ rRNA sequencing data, we use a $16 \mathrm{~S}$ simulator sparseDOSSA [89] to generate the abundances of 150 taxa in 100 samples under two conditions (see Additional file 1: Simulation 4). Among these 150 taxa, 45 are predefined as truly DA taxa. We apply five state-of-the-art DA methods: the Wilcoxon rank-sum test, ANCOM [27], metagenomeSeq [35], DESeq2-phyloseq [33, 34], and Omnibus test [102]. To evaluate the accuracy of DA taxon identification, we calculate the precision, recall, and $\mathrm{F}_{1}$ score (i.e., the harmonic mean of precision and recall) of each method, with or without using mbImpute as a preceding step, by comparing each method's detected DA taxa to the truly DA taxa. Note that metagenomeSeq uses the 
zero-inflated Gaussian linear model for log-transformed microbiome data, but this model does not fit well to imputed data, which have many zeros removed; hence, we use the Gaussian linear model without zero-inflation to evaluate metagenomeSeq on imputed data. Under the false discovery rate (FDR) thresholds of 0.05 (Fig. 2e) and 0.1 (Additional file 1: Figure S4), the mbImpute-empowered DA methods consistently have better recall rates and $F_{1}$ scores than those of the same DA methods without imputation. Notably, mbImpute improves both precision and recall rates of metagenomeSeq.

To evaluate the robustness of mbImpute to sequencing depth, we simulate 16S rRNA sequencing data based on real data for 300 taxa in 54 samples with four sequencing depths: 1000, 2000, 5000, and 10,000 reads per sample (see Additional file 1: Simulation 5). Additional file 1: Figure S5a shows that mbImpute has better imputation accuracy as sequencing depth increases. This is an expected result because a larger sequencing depth leads to fewer missing data so that mbImpute can be better trained with more non-missing data. We further evaluate the performance of the five non-microbiome imputation methods along with mbImpute. Additional file 1: Figure S6 shows that softImpute and ALRA, the two low-rank matrix factorization methods, also have better imputation accuracy as sequencing depth increases, yet their accuracies are worse than those of mbImpute at all sequencing depths. Unexpectedly, the four other imputation methods developed for scRNA-seq data-SAVER, scImpute, MAGIC, and ALRA-show no improvement over the baseline, "no imputation." One possible reason is that the sequencing depths used in this simulation $\left(\sim 10^{3}\right)$ are much lower than those of typical scRNA-seq data $\left(\sim 10^{6}\right)$. These results again suggest that scRNA-seq imputation methods are unsuitable for microbiome $16 \mathrm{~S}$ rRNA sequencing data. We also check the robustness of mbImpute to outlier samples. Taking the sample with the 2000-read persample sequencing depth, we generate one or two outlier samples by assigning large abundance values to 62 lowly abundant taxa in the existing 54 samples and setting other taxa's abundance to zero (see Additional file 1: Simulation 5). Additional file 1: Figure S5b shows that the imputation accuracy of mbImpute is robust to the introduction of outlier samples. Additional file 1: Figure S7 shows the abundance distributions of four example taxa with outlier values before and after imputation. We observe that the existence of outliers does not distort the post-imputation distribution of non-outlier samples.

\section{mblmpute empowers DESeq2-phyloseq in DA taxon analysis}

We find that mbImpute works well with DESeq2-phyloseq [33, 34], a widely used DA method for microbiome data, on real WGS datasets. We perform DA analysis on two T2D WGS datasets $[18,19]$ and four CRC WGS datasets [14-17], with or without using mbImpute as a preceding step. The goal of DA analysis is to identify the DA taxa between the diseased and control samples. These DA taxa may serve as potential targets for early detection or treatment of disease [14]. Note that mbImpute does not utilize the samples' group information (whether each sample belongs to the diseased or control group) for its imputation, so that mbImpute will not falsely increase sample similarity within groups.

We start with the five DA methods-Wilcoxon rank-sum test, ANCOM, metagenomeSeq, DESeq2-phyloseq, and Omnibus test-for identifying disease-related DA taxa in the two T2D and four CRC datasets. Under the FDR threshold 0.05, only DESeq2-phyloseq and Omnibus test identify DA taxa in all datasets (Additional file 1: Table S1). Hence, we focus on evaluating the accuracy of DESeq2-phyloseq and Omnibus test on the orig- 
inal and imputed data (for DESeq2-phyloseq applied to the imputed data, we refer to it as mbImpute-empowered DESeq2-phyloseq). For a sanity check on the DA taxon identification results in each dataset, we plot the distribution of taxa's $p$ values calculated by DESeq2-phyloseq or Omnibus test before and after mbImpute is applied (Additional file 1: Figures S8-9). We find that all the $p$ value distributions for DESeq2-phyloseq match our expectation (i.e., the expected $p$ value distribution should have a mode near zero and be uniform elsewhere). However, the $p$ value distributions for Omnibus test exhibit abnormality for the Karlsson et al. T2D dataset [18] and Vogtmann et al. CRC dataset [17]. Specifically, the distributions have an unexpected mode near one for the Karlsson et al. T2D dataset [18] after imputation and for the Vogtmann et al. CRC dataset [17] before and after imputation. This phenomenon suggests that the distributional assumption of Omnibus test does not hold for these data. Hence, we focus on the comparison between DESeq2-phyloseq and mbImpute-empowered DESeq2-phyloseq in the following analysis.

To investigate whether the DA taxa identified by DESeq2-phyloseq or mbImputeempowered DESeq2-phyloseq are meaningful disease markers, we evaluate the predictive power of the identified DA taxa for sample disease conditions (control or diseased). For each microbiome dataset, we use the DA taxa, identified by DESeq2-phyloseq or mbImpute-empowered DESeq2-phyloseq, as features and apply the random forest algorithm to predict sample disease conditions. We use the 5 -fold cross-validated precisionrecall area under the curve (PR-AUC) to evaluate the prediction accuracy (Fig. 3a). We observe that mbImpute-empowered DESeq2-phyloseq leads to overall better prediction accuracy than DESeq2-phyloseq does across the six datasets.

Then, we focus on the Karlsson et al. T2D dataset [18] and the Vogtmann et al. CRC dataset [17], which exhibit the largest improvement in prediction accuracy when the DA taxa identified by mbImpute-empowered DESeq2-phyloseq are used. For the Karlsson et al. T2D dataset [18], we observe that mbImpute-empowered DESeq2-phyloseq outputs a greater number of small $p$ values than DESeq2-phyloseq does (Additional file 1: Figure S7), suggesting that more taxa are identified as DA after imputation (in fact, all the DA taxa identified before imputation are still found as DA after imputation). Hence, the improvement in prediction accuracy implies that the DA taxa identified only after imputation contribute to the distinction between control and T2D samples. In particular, we examine three example taxa (Ruminococcus species) identified as DA only after imputation. Figure $3 \mathrm{~b}$ shows the distributions of these three taxa's abundances (on the log-scale) before and after imputation. For each taxon, we observe that the imputed abundances and the original non-zero abundances have similar ranges and both suggest that the taxon is more abundant in T2D samples than in control samples. However, this abundance difference is obscured by the prevalent zeros before imputation and thus cannot be captured by DESeq2-phyloseq. Literature evidence is consistent with the post-imputation result of the first two taxa. Specifically, the first taxon, Ruminococcus sp_5_1_39BFAA, has decreased abundances in T2D patients after the Acarbose treatment [103]. The second taxon, Ruminococcus callidus, is shown to be enriched in T2D mouse models [104].

For the Vogtmann et al. CRC dataset [17], the 5-fold cross-validated PR-AUC increases by almost $10 \%$ when the DA taxa identified after imputation, instead of those identified before imputation, are used as features. In fact, fewer taxa are identified as DA after imputation (Additional file 1: Figure S8). At the $q$ value threshold 0.05, DESeq2- 


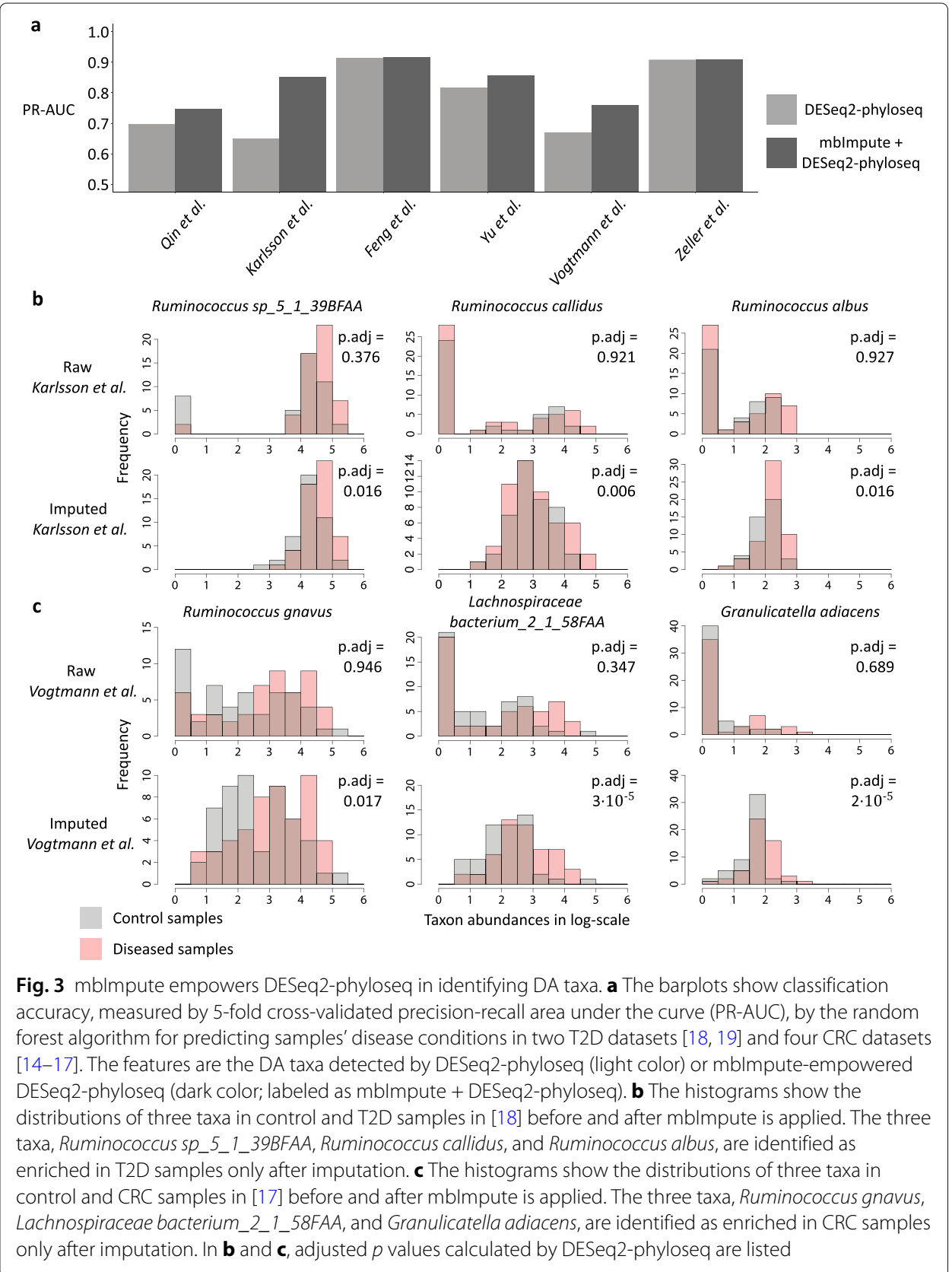

phyloseq identifies 53 DA taxa, while mbImpute-empowered DESeq2-phyloseq identifies 40 DA taxa, with only 17 taxa in overlap. This result suggests that the 23 DA taxa identified only after imputation contribute much to the distinction between control and CRC samples. We examine three of these 23 taxa: Ruminococcus gnavus, Lachnospiraceae bacterium_2_1_58FAA, and Granulicatella adiacens. Figure 3c shows that each taxon has its imputed abundances and its original non-zero abundances in similar ranges; its imputed and original non-zero abundances both suggest it to be more abundant in CRC samples than in control samples. However, this abundance difference is obscured by the prevalent zero abundances before imputation and thus cannot be captured by DESeq2-phyloseq. To confirm the post-imputation result, we find literature evidence for the three taxa. 
First, several studies have reported that Ruminococcus gnavus is associated with a higher risk of CRC [99, 105-107]. Second, two studies have shown that Lachnospiraceae bacterium_2_1_58FAA is positively associated with colorectal neoplasms, from which CRC arises [99]. Third, Granulicatella adiacens is reported to be associated with CRC progression in both human [83] and mouse studies [108]. We also examine the taxa identified as DA before imputation but not as DA after imputation, and we find that these taxa only differ in zero proportions and have similar non-zero abundance distributions between control and CRC samples (Additional file 1: Figure S10). We argue that such taxa are unlikely to be truly DA because it is questionable whether zero proportion differences are biologically meaningful given the prevalence of technical and sampling zeros. Together, our analysis results on the Karlsson et al. T2D dataset [18] and the Vogtmann et al. CRC dataset [17] suggest that compared to DESeq2-phyloseq, mbImpute-empowered DESeq2-phyloseq can detect DA taxa that are more predictive of sample conditions, and we verify that some DA taxa only detected by mbImpute-empowered DESeq2-phyloseq are functionally relevant by literature evidence.

For all the DA taxa identified by DESeq2-phyloseq and mbImpute-empowered DESeq2phyloseq in the two T2D and four CRC data datasets, we query the GMrepo database [99] and find two T2D- and one CRC-related functional terms. For each term, we perform the Fisher's exact test to check its enrichment in the DA taxa identified from the corresponding disease-related datasets. Our results show that all three terms are more enriched in the DA taxa identified after mbImpute is applied (Table 1; Additional files 1,2, $3,4,5,6$ and 7), providing functional support to the efficacy of mbImpute in empowering DESeq2-phyloseq.

Furthermore, we analyze the overlap of the DA taxa identified in the two T2D datasets $[18,19]$. There is no overlap in the two sets of DA taxa identified by DESeq2-phyloseq, but Clostridium bolteae is identified by mbImpute-empowered DESeq2-phyloseq in both datasets. In fact, Clostridium bolteae has been reported as enriched in T2D samples in the Qin et al. dataset [19] but not in the Karlsson et al. dataset [18] In our analysis on the Karlsson et al. T2D dataset [18], Clostridium bolteae has FDR-adjusted $p$ values 0.347 and 0.036 before and after imputation, respectively (abundance distributions in Additional file 1: Figure S11). Literature evidence suggests that Clostridium bolteae is positively associated with T2D in both human [109] and mouse studies [110].

For the four CRC datasets [14-17], we analyze the DA taxa identified in at least two datasets before and after imputation. Specifically, DESeq2-phyloseq and mbImputeempowered DESeq2-phyloseq respectively identify four and 18 taxa (with three taxa in

Table 1 Fisher's exact test $p$ values about the enrichment of T2D- and CRC-related functional terms in the DA taxa found by DESeq2-phyloseq or mblmpute-empowered DESeq2-phyloseq

\begin{tabular}{llll}
\hline DA method & T2D term 1* & T2D term 2** & CRC term*** \\
\hline DESeq2-phyloseq & 0.54 & 0.76 & 0.0027 \\
mblmpute-empowered DESeq2-phyloseq & 0.03 & 0.17 & 0.0010 \\
\hline
\end{tabular}

For each term, the DA taxa identified by each method from the corresponding datasets are pooled to do the test.

*T2D term 1: "The time period before the development of symptomatic diabetes. For example, certain risk factors can be observed in subjects who subsequently develop INSULIN RESISTANCE as in type 2 diabetes (DIABETES MELLITUS, TYPE 2)." **T2D term 2: "A cluster of symptoms that are risk factors for CARDIOVASCULAR DISEASES and TYPE 2 DIABETES MELLITUS. The major components of metabolic syndrome include ABDOMINAL OBESITY; atherogenic DYSLIPIDEMIA; HYPERTENSION; HYPERGLYCEMIA; INSULIN RESISTANCE; a proinflammatory state; and a prothrombotic (THROMBOSIS) state."

${ }^{* * *}$ CRC term: "Tumors or cancer of the COLON or the RECTUM or both. Risk factors for colorectal cancer include chronic ULCERATIVE COLITIS; FAMILIAL POLYPOSIS COLI; exposure to ASBESTOS; and irradiation of the CERVIX UTERI" 
overlap) that have significantly lower abundances in CRC samples than in normal samples. Among these taxa, DESeq2-phyloseq only identifies Bifidobacterium animalis, while mbImpute-empowered DESeq2-phyloseq additionally identifies three other Bifidobacterium species: Bifidobacterium bifidum, Bifidobacterium catenulatum, and Bifidobacterium longum. Additional file 1: Figures S12-14 show the distributions of these three taxa's abundances (on the log-scale) before and after imputation. Literature evidence indicates that Bifidobacterium is beneficial to the immune system against CRC [111-113] and has been used as probiotics [114]; all the four Bifidobacterium species detected by mbImpute-empowered DESeq2-phyloseq have been reported to have significantly lower abundances in CRC samples $[115,116]$. Together, our overlap analysis on T2D and CRC datasets suggests that mbImpute helps recover the DA taxa that are detected in one dataset but missed in another due to prevalent zeros.

\section{mblmpute preserves distributional characteristics of taxa's non-zero abundances and recovers downsampling zeros}

In the DA analysis described in the last section, we observe that mbImpute can well maintain the distributions of taxa's non-zero abundances, see Fig. 3b, c. To further verify the property of mbImpute in preserving characteristics of non-zero abundances, we examine pairwise taxon-taxon relationships in the two T2D WGS datasets: Karlsson et al. and Qin et al. datasets $[18,19]$. For a pair of taxa, we calculate two Pearson correlations based on the raw data on the log-scale: one using all the samples ("raw all-sample correlation") and the other using only the samples where both taxa have non-zero abundances ("raw non-zero-sample correlation"). In this section, we perform our analysis on the log-scale of the taxa count matrix since one of the assumptions for Pearson correlation is the normality of both variables, and microbiome count data on the log-scale better resemble a continuous normal distribution. For the same pair of taxa, we also calculate a Pearson correlation based on the imputed data by mbImpute on the logscale, using all the samples ("imputed all-sample correlation"). As shown in Fig. 4a, b, there are vast differences between the raw all-sample correlations and the corresponding raw non-zero-sample correlations. However, the imputed all-sample correlations better resemble the corresponding raw non-zero-sample correlations, suggesting that mbImpute well preserves pairwise taxon-taxon correlations encoded in taxa's non-zero abundances.

We also explore the linear relationship of each taxon pair using the standard major axis (SMA) regression, which, unlike the least-squares regression, treats two taxa symmetrically. For a pair of taxa, we perform two SMA regressions on the raw data: one using all the samples ("raw all-sample regression") and the other using only the samples where both taxa have non-zero abundances ("raw non-zero-sample regression"). We also perform the SMA regression on the imputed data by mbImpute, using all the samples ("imputed all-sample regression"). Figure 4a, b show that the raw all-sample regressions and the raw non-zero-sample regressions return vastly different lines. Especially, the two lines between Eubacterium sirasum and Ruminococcus obeum in the Karlsson et al. T2D dataset [18] (Fig. 4b bottom left) have slopes with opposite signs. In contrast, the imputed all-sample regressions output lines with slopes similar to those of the raw nonzero-sample regressions. This result again confirms mbImpute's capacity for preserving characteristics of taxa's non-zero abundances in microbiome data. 


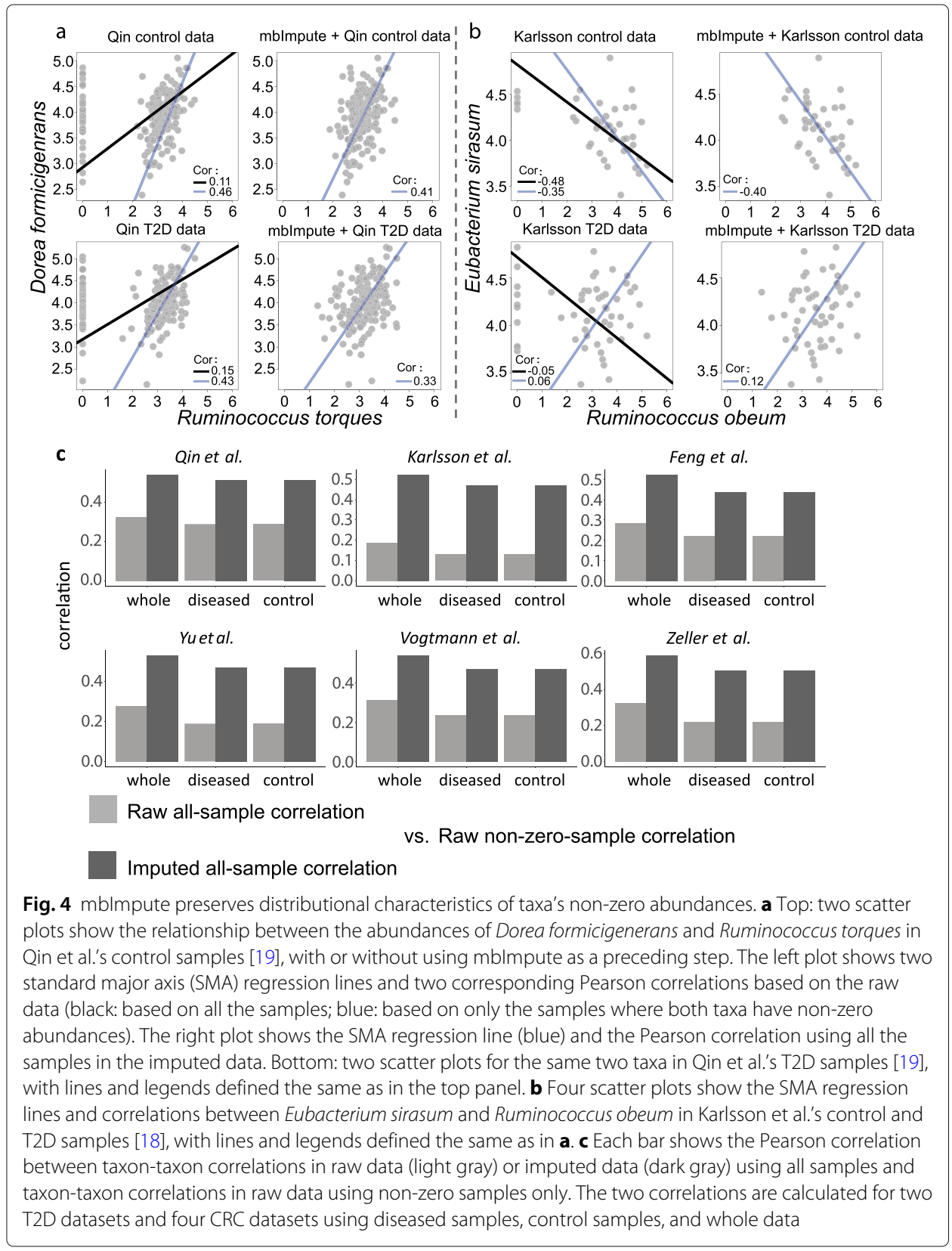

Furthermore, we systematically evaluate the performance of mbImpute in preserving raw non-zero-sample correlations on the two T2D WGS datasets and the four CRC WGS datasets, with each dataset containing samples in two groups: diseased and control. Figure $4 \mathrm{c}$ show that the imputed all-sample correlations resemble the raw non-zerosample correlations much better than the raw all-sample correlations do, on every dataset including all samples ("whole" in Fig. 4c). Moreover, within each sample group in each dataset ("diseased" and "control" in Fig. 4c), the imputed all-sample correlations still better resemble the raw non-zero-sample correlations than the raw all-sample correlations do. Note that the resemblance is defined based on the Pearson correlation of two sets of 
correlations. Additional file 1: Figure S15 shows that the same conclusion holds when the resemblance is defined based on the Spearman correlation. Note that mbImpute does not use the group information of each sample in its imputation process.

Our results echo existing concerns about spurious taxon-taxon correlations in microbiome data due to excess non-biological zeros $[117,118]$. In other words, taxon-taxon correlations cannot be accurately estimated from raw data using all samples. Without imputation, an intuitive approach is to use taxa's non-zero abundances to estimate taxontaxon correlations; however, this approach reduces the sample size for estimating each taxon pair's correlation because it does not use the samples containing zero abundances for either taxon, and it also makes different taxon pairs' correlation estimates rely on different samples. To address these issues, mbImpute provides another approach: its imputed data allow taxon-taxon correlations to be estimated from all samples. Moreover, we observe that mbImpute makes log-transformed taxon abundances closer to be normally distributed (Additional file 1: Figure S16); thus, the Pearson correlation is a more meaningful measure for taxon-taxon associations on the imputed data than on the raw data.

In addition, based on the T2D WGS dataset generated by Qin et al. [19], we verify mbImpute's capacity to identify non-biological zeros generated by downsampling. In each sample (i.e., each row in the sample-by-taxon count matrix), we assign every taxon a sampling probability proportional to its count, i.e., the larger the count, the more likely the taxon is to be sampled; based on these probabilities, we sample $60 \%$ or $30 \%$ of the nonzero taxon counts, and we set the unsampled counts to zeros (corresponding to a removal rate of $40 \%$ or $70 \%$ ); we repeat the downsampling independently for ten times. After applying mbImpute to the downsampled count matrices, we find that mbImpute correctly identifies $95.83 \%$ and $92.83 \%$ (on average) of the newly introduced non-biological zeros under the two removal rates. Before imputation, the average Pearson correlations between the downsampled matrices and the original matrix (on the log-scale) are 0.76 and 0.53 under the two removal rates. After applying mbImpute to all the three matrices, the correlations are increased to 0.87 and 0.76 (Table 2). This result confirms the effectiveness of mbImpute in recovering zeros due to downsampling.

\section{mblmpute increases the similarity of microbial community structure between $16 \mathrm{~S}$ rRNA and WGS data}

We further show that mbImpute can enhance the similarity of taxon-taxon correlations inferred from microbiome data measured by two technologies-16S rRNA sequencing

Table 2 Effectiveness of mblmpute in identifying zeros due to downsampling of Qin et al.'s T2D WGS dataset [19]. For each of two removal rates $40 \%$ and $70 \%$, we repeat independent downsampling for ten times

\begin{tabular}{lll}
\hline Removal rate & $\mathbf{4 0 \%}$ & $\mathbf{7 0 \%}$ \\
\hline \% of downsampling zeros identified & $95.83 \% \pm 0.46 \%$ & $92.83 \% \pm 0.92 \%$ \\
Pearson correlation before imputation & $0.7565 \pm 0.0023$ & $0.5261 \pm 0.0016$ \\
Pearson correlation after imputation & $0.8747 \pm 0.0100$ & $0.7582 \pm 0.0235$ \\
\hline
\end{tabular}

For each removal rate (column), the first row lists the average percentage of downsampling zeros identified by mblmpute; the second row lists the average Pearson correlation between a downsampled matrix and the original matrix (on the log-scale) before imputation; the third row lists the average Pearson correlation (on the log-scale) after mblmpute is used. Each average calculated across the ten downsampling and is accompanied with an error margin, i.e., 1.96 times the standard error over the ten downsampling 
and WGS. We use two microbiome datasets of healthy human stool samples: a 16S rRNA dataset from the Human Microbiome Project [119] and a WGS dataset from the control samples in Qin et al. [19] We compare the genus-level taxon-taxon correlations between these two datasets, and we perform the comparison again after applying mbImpute. Figure 5 shows that mbImpute increases the similarity between the taxon correlation structures in the two datasets. Before imputation, the Pearson correlation between the two correlation matrices (one computed from 16S rRNA taxon abundances and the other from WGS taxon abundances) is 0.59 ; mbImpute increases the correlation to 0.64 . In particular, we observe three taxon groups (highlighted by magenta, green, and purple squares in Fig. 5) supported by both $16 \mathrm{~S}$ rRNA and WGS data after imputation. Notably, in the magenta squares, Acidaminococcus has correlations with Dialister and Blautia only after

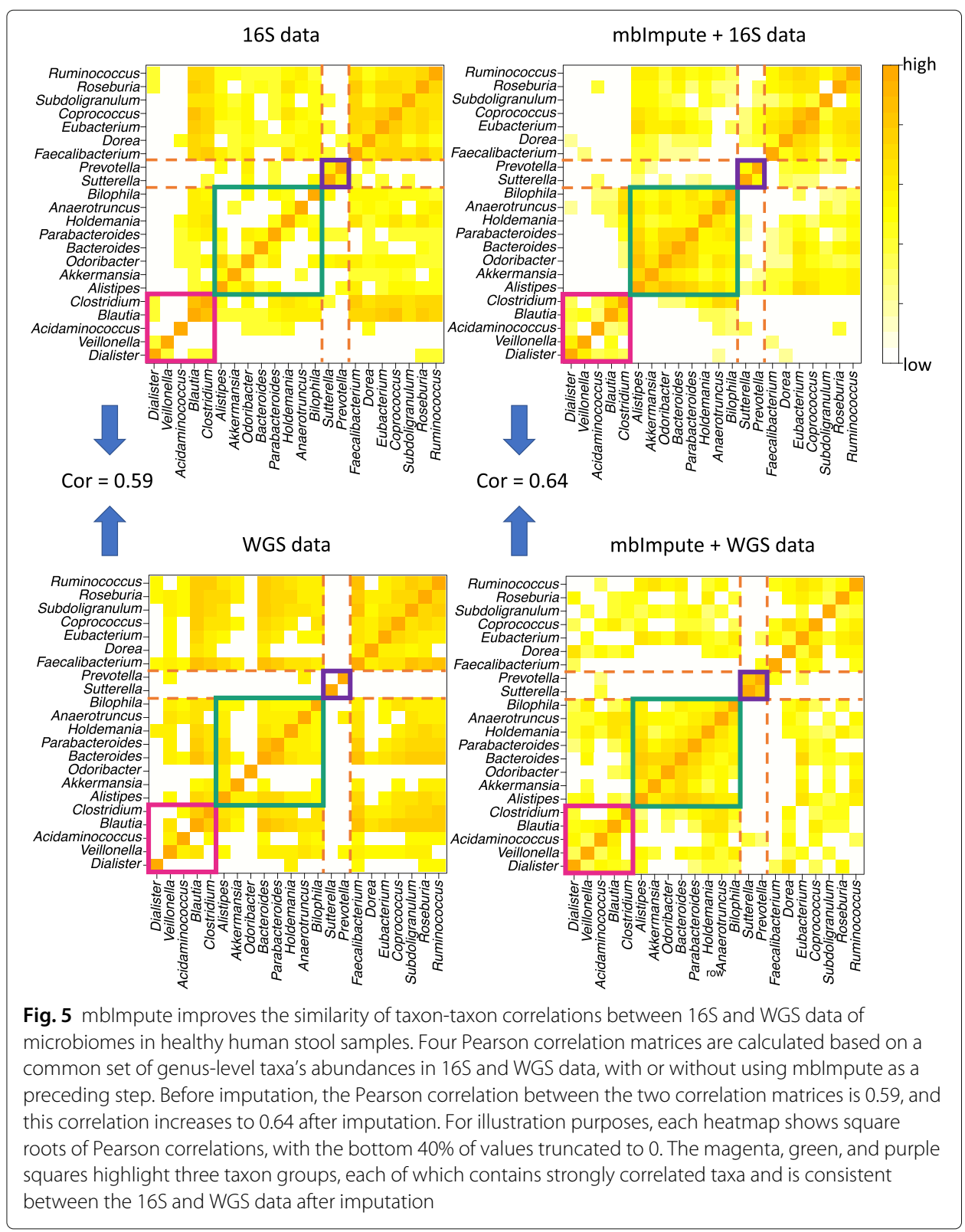


imputation, and this result is consistent with the literature: Acidaminococcus and Dialister are both reported to have low abundances in healthy human stool samples [120]; Acidaminococcus and Blautia are both associated with risks of T2D and obesity, lipid profiles, and homeostatic model assessment of insulin resistance [121]. The green squares contain three bile-tolerant genera: Alistipes, Bilophila, and Bacteroides [122]. The raw $16 \mathrm{~S}$ and WGS data only reveal the correlation between Bacteroides and Alistipes, but mbImpute recovers the correlations Bilophila has with Alistipes and Bacteroides. The purple squares indicate a strong correlation between Sutterella and Prevotella after imputation, yet this correlation is not observed in raw WGS data. We verify this correlation in the MACADAM database [123], which contains metabolic pathways associated with microbes. Out of 1260 pathways, Sutterella and Prevotella are associated with 154 and 278 pathways, respectively, and 122 pathways are in common; Fisher's exact test finds that the overlap is statistically significant ( $p$ value $<2.2 \times 10^{-16}$ ), suggesting that Sutterella and Prevotella may be functionally related. Overall, our results indicate that mbImpute can facilitate meta-analysis of $16 \mathrm{~S}$ and WGS data by alleviating the hurdle of prevalent non-biological zeros.

We perform a negative control study to confirm that the increased similarity between 16S rRNA and WGS data is not an artifact introduced by mbImpute. We use a 16S rRNA dataset of human oral samples and a WGS dataset of human stool samples, which are expected to have different genus-level taxon-taxon correlations. Same as in the previous study, we compare the genus-level taxon-taxon correlations between the two datasets before and after applying mbImpute. Additional file 1: Figure S17 shows that mbImpute decreases the similarity between the taxon correlation matrices of the two datasets. Before imputation, the Pearson correlation between the two correlation matrices is 0.21 ; mbImpute decreases the correlation to 0.09 .

\section{Discussion}

A critical challenge in microbiome data analysis is statistical inference of taxon abundance from highly sparse and noisy data. Our proposed method, mbImpute, will address this challenge and facilitate analysis of both $16 \mathrm{~S}$ and WGS data; mbImpute works by correcting non-biological zeros and retaining taxa's non-zero abundance distributions after imputation. As the first imputation method designed for microbiome data, mbImpute is shown to outperform multiple state-of-the-art imputation methods developed for other data types. In the DA analysis, we show that mbImpute-empowered DESeq2-phyloseq has better performance in selecting predictive taxa for disease conditions comparing to DESeq2-phyloseq. The reason is that mbImpute-empowered DESeq2-phyloseq is able to identify the taxa missed by the DESeq2-phyloseq (due to excess zeros) but should be called DA (i.e., having non-zero abundances that exhibit significantly different means between two sample groups). We then demonstrate that mbImpute preserves taxa's non-zero abundance distributions. As a result, taxon-taxon correlations calculated from all samples after imputation better resemble the taxon-taxon correlations calculated from non-zero counts only. Hence, mbImpute can facilitate taxon network analysis by allowing all taxon pairs to have meaningful correlations computed from all samples. Moreover, mbImpute improves the reproducibility of DA taxon identification across studies and the consistency of microbial community detection between $16 \mathrm{~S}$ and WGS data. 
In the application of mbImpute, two practical concerns are what normalization method and phylogenetic distance metric work the best with mbImpute. First, the goal of normalization is to make taxon counts comparable across samples, a necessary assumption of mbImpute. In our results, we think our way of normalization is sufficient to meet this assumption. However, the appropriate normalization method for mbImpute is case by case in future applications, depending on whether confounders such as batch effects are observable; hence, users' judgment is indispensable. We recognize that benchmarking normalization methods for microbiome data is a separate project. Hence, we refer users to benchmark papers [20,124] to guide their choice of benchmark methods. Second, users may specify the phylogenetic distances between taxa based on their domain knowledge. In our results, we define the phylogenetic distance between two taxa as the number of branches connecting them in a phylogenetic tree, but alternative choices exist, such as the total lengths of the branches. If users want to choose a distance metric, we recommend that they supply the phylogenetic distances defined by candidate metrics into mbImpute and choose the metric that leads to the smallest cross-validated MSE, i.e., the cross-validated imputation error of mbImpute on non-missing data.

Regarding the mbImpute-empowered DA analysis, we note that it offers a new perspective of identifying DA taxa from microbiome 16S and WGS data after imputation. We have summarized three statistical definitions of DA taxa in microbiome data in Additional file 1: Statistical definitions of DA taxa. Note that mbImpute-empowered DA analysis is advantageous in that it alleviates the existence of non-biological zeros, and it uses all available samples for DA testing. A controversial question is, if a taxon has few zeros in condition 1 but few non-zeros in condition 2, and the non-zero values have similar magnitudes in the two conditions, whether or not should this taxon be called DA. We note that mbImpute is unlikely to impute the predominant zeros in condition 2 because it would treat these zeros as biological zeros. Hence, mbImpute-empowered DA analysis is likely to call such a taxon as DA.

There has been a long-standing concern about sample contamination in microbiome sequencing data, e.g., contamination from DNA extraction kits and laboratory reagents $[125,126]$. Existing studies have attempted to address this issue via calibrated sequencing operations [126-128] and computational methods [129, 130]. We recommend researchers to perform contamination removal before applying mbImpute. Moreover, by its design, mbImpute is robust to certain types of sample contamination that result in outlier taxa and samples. For each outlier taxon, mbImpute would borrow little information from other taxa to impute this outlier taxon's abundances. Similarly, mbImpute is robust to the existence of outlier samples that do not resemble any other sample.

In statistical inference, a popular and powerful technique is the use of indirect evidence by borrowing information from other observations, as seen in regression, shrinkage estimation, empirical Bayes, among many others [131]. Imputation follows the indirect evidence principle, where the most critical issue is to decide what observations to borrow information from so as to improve data quality instead of introducing excess biases. To achieve this, mbImpute employs penalized regression to selectively leverage similar samples, similar taxa, and sample covariates to impute likely non-biological zeros, whose identification also follows the indirect evidence principle by incorporating sample covariates into consideration. Also, mbImpute provides a flexible framework to make use of 
microbiome metadata: it selectively borrows metadata information when available, but it does not rely on the existence of metadata (see Methods).

In the comparison of mbImpute with softImpute, a general matrix imputation method widely used in other fields, we observe that softImpute's imputed taxon abundances exhibit artificial spikes and smaller variances than those of the original non-zero abundances, possibly due to its low-rank assumption. In contrast, mbImpute is a regressionbased method that focuses more on local matrix structures, and we find that it retains well the original non-zero abundance distributions. We will investigate the methodological differences between mbImpute and softImpute in a future study.

Moreover, we observe that, similar to each taxon's non-zero abundances, the imputed abundances exhibit a bell-shaped distribution across samples on the log-scale. This suggests that statistical methods utilizing normal distributional assumptions become suitable and applicable to imputed taxon abundances. A possible use of imputed microbiome data is to construct a taxon-taxon interaction network, to which network analysis methods may be applied to find taxon modules and hub taxa [132]. As a preliminary exploration, we construct Bayesian networks of taxa based on the two T2D datasets [18, 19] after applying mbImpute. Interesting changes are observed in taxon interactions from control samples to T2D samples (Additional file 1: Figures S18-19). For example, two genera, Ruminococcus and Eubacterium, have interactive species in control samples but not in T2D samples. In future research, differential network analysis methods can be applied to find taxon communities that differ between two sample groups.

\section{Methods}

\section{mblmpute methodology}

Here, we describe mbImpute, a statistical method that corrects prevalent non-biological zeros in microbiome data. As an overview, mbImpute takes a taxon count matrix as input; pre-processes the data; identifies the likely non-biological zeros and imputes them based on the input count matrix, sample covariates, and taxon phylogeny; and outputs an imputed count matrix.

\section{Notations}

We denote the sample-by-taxon taxa count matrix as $\mathbf{M}=\left(M_{i j}\right) \in \mathbb{Z}_{\geq 0}^{n \times m}$, where $n$ is the number of microbiome samples and $m$ is the number of taxa. We denote the sample covariate matrix (i.e., metadata) as $\mathbf{X} \in \mathbb{R}^{n \times q}$, where $q$ equals the number of covariates plus one (for the intercept). (By default, mbImpute includes sample library size as a covariate.) In addition, we define a phylogenetic distance matrix of taxa as $\mathbf{D}=\left(D_{j j^{\prime}}\right) \in \mathbb{Z}_{\geq 0}^{m \times m}$, where $D_{j j^{\prime}}$ represents the number of branches connecting taxa $j$ and $j^{\prime}$ in the phylogenetic tree or user-specified distance between taxa $j$ and $j^{\prime}$.

\section{Data pre-processing}

mbImpute requires every taxon's counts across samples to be on the same scale before imputation. If this condition is unmet, normalization is needed. However, how to properly normalize microbiome data is challenging, and multiple normalization methods have been developed in recent years $[29,133,134]$. Regarding the choice of an appropriate normalization method, users may refer to benchmark papers [20, 124]. To give users the flexibility of choosing an appropriate normalization method, mbImpute allows users 
to input a normalized count matrix by specifying that the input matrix does not need normalization. Otherwise, mbImpute normalizes samples by library size.

Default normalization (optional) To account for the varying library sizes (i.e., total counts) of samples, mbImpute first normalizes the count matrix $\mathbf{M}$ by row. The normalized count matrix is denoted as $\mathbf{M}^{(\mathcal{N})}=\left(M_{i j}^{(\mathcal{N})}\right) \in \mathbb{R}_{\geq 0}^{n \times m}$, where

$$
M_{i j}^{(\mathcal{N})}=10^{6} \cdot \frac{M_{i j}}{\sum_{j^{\prime}=1}^{m} M_{i j^{\prime}}} .
$$

After this normalization, every sample has a total count of $10^{6}$.

mbImpute applies the logarithmic transformation to the normalized counts so as to reduce the effects of extremely large counts [82]. The resulted log-transformed normalized matrix is denoted as $\mathbf{Y}=\left(Y_{i j}\right) \in \mathbb{R}_{>0}^{n \times m}$, with

$$
Y_{i j}=\log _{10}\left(M_{i j}^{(\mathcal{N})}+1.01\right),
$$

where the value 1.01 is added to make $Y_{i j}>0$ to avoid the occurrence of infinite values in a later parameter estimation step, following $[50,81]$. This logarithmic transformation allows us to fit a continuous probability distribution to the transformed data, thus simplifying the statistical modeling. In the following text, we refer to $\mathbf{Y}$ as the sample-by-taxon abundance matrix.

\section{mblmpute step 1: identification of taxon abundances that need imputation}

mbImpute assumes that each taxon's abundances, i.e., a column in $\mathbf{Y}$, follow a mixture model. The model consists of two components: a Gamma distribution for the taxon's likely non-biological zeros and low abundances and a normal distribution for the taxon's actual abundances, with the normal mean incorporating sample covariate information (including sample library size as a covariate). Specifically, mbImpute assumes that the abundance of taxon $j$ in sample $i, Y_{i j}$, follows the following mixture distribution:

$$
Y_{i j} \sim p_{j} \cdot \Gamma\left(\alpha_{j}, \beta_{j}\right)+\left(1-p_{j}\right) \cdot \mathcal{N}\left(X_{i \cdot \gamma}^{\top} \gamma_{j}, \sigma_{j}^{2}\right),
$$

where $p_{j} \in(0,1)$ is the missing rate of taxon $j$, i.e., the probability that taxon $j$ is falsely undetected, $\Gamma\left(\alpha_{j}, \beta_{j}\right)$ denotes the Gamma distribution with shape parameter $\alpha_{j}>0$ and rate parameter $\beta_{j}>0$, and $\mathcal{N}\left(X_{i}^{\top} \gamma_{j}, \sigma_{j}^{2}\right)$ denotes the normal distribution with mean $X_{i}^{\top} \gamma_{j}$ and standard deviation $\sigma_{j}>0$. In other words, with probability $p_{j}, Y_{i j}$ is a missing value that needs imputation; with probability $1-p_{j}, Y_{i j}$ is sampled from the non-missing abundance distribution of taxon $j$ and does not need imputation. mbImpute models the normal mean parameter as a linear function of sample covariates: $X_{i}^{\top} \gamma_{j}$, where $X_{i} \in \mathbb{R}^{q}$ denotes the $i$ th row in the covariate matrix $\mathbf{X}$, i.e., the covariates of sample $i$, and $\gamma_{j} \in \mathbb{R}^{q}$ represents the $q$ covariates' effects (including the intercept) on taxon $j$ 's abundance. This formulation allows a taxon to have similar expected abundances (when not missing) in samples with similar covariates.

The intuition behind this model is that taxon $j$ 's non-missing abundance in a sample is drawn from a normal distribution, whose mean depicts the expected abundance given the sample covariates. However, due to library preparation and under-sampling issues in sequencing, false zero or low counts could have been introduced into the data, creating another mode near zero in taxon $j$ 's abundance distribution. mbImpute models that mode using a Gamma distribution with mean $\alpha_{j} / \beta_{j}$, which is close to zero. 
mbImpute fits this mixture model to taxon $j$ 's abundances using the expectationmaximization (EM) algorithm to obtain the maximum likelihood estimates $\hat{p}_{j}, \hat{\alpha}_{j}, \hat{\beta}_{j}, \hat{\gamma}_{j}$, and $\hat{\sigma}_{j}^{2}$. Additional file 1: Figure S20 shows four examples where the fitted mixture model well captures the bimodality of an individual taxon's abundance distribution. However, some taxa are observed to have an abundance distribution containing a single mode that can be well modeled by a normal distribution. When that occurs, the EM algorithm would encounter a convergence issue. To fix this, mbImpute uses a likelihood ratio test (LRT) to first decide if the Gamma-normal mixture model fits to taxon $j$ 's abundances significantly better than a normal distribution $Y_{i j} \sim \mathcal{N}\left(X_{i}^{\top} \eta_{j}, \omega_{j}^{2}\right)$ does. Given the maximum likelihood estimates $\hat{\eta}_{j}$ and $\hat{\omega}_{j}^{2}$ and under the assumption that $Y_{i j}$ 's are all independent, the LRT statistic of $\operatorname{taxon} j$ is:

$$
\Lambda_{j}=-2 \ln \frac{\prod_{i=1}^{n} f_{\mathcal{N}}\left(Y_{i j} ; X_{i}^{\top}, \hat{\eta}_{j}, \hat{\omega}_{j}^{2}\right)}{\prod_{i=1}^{n}\left[\hat{p}_{j} \cdot f_{\Gamma}\left(Y_{i j} ; \hat{\alpha}_{j}, \hat{\beta}_{j}\right)+\left(1-\hat{p}_{j}\right) \cdot f_{\mathcal{N}}\left(Y_{i j} ; X_{i}^{\top} \hat{\gamma}_{j}, \hat{\sigma}_{j}^{2}\right)\right]},
$$

which asymptotically follows a chi-square distribution with 3 degrees of freedom (because the mixture model has three more parameters than in the normal model) under the null hypothesis that the normal model is the correct model. We summarize the LRT $p$ values calculated on six real WGS datasets and observe that few taxa have $p$ values greater than 0.05 (see Additional file 1: Figure S21a). Additional file 1: Figure S21b shows the distribution of one randomly picked taxon with LRT $p$ value greater than 0.05 in each dataset; these taxa's log-transformed counts do not have a mode close to zero. If the LRT $p$ value $\leq 0.05$, mbImpute uses the mixture model to decide which abundances of taxon $j$ need imputation. Specifically, mbImpute decides if $Y_{i j}$ needs imputation based on the estimated posterior probability that $Y_{i j}$ comes from the Gamma component:

$$
d_{i j}=\frac{\hat{p}_{j} \cdot f_{\Gamma}\left(Y_{i j} ; \hat{\alpha}_{j}, \hat{\beta}_{j}\right)}{\hat{p}_{j} \cdot f_{\Gamma}\left(Y_{i j} ; \hat{\alpha}_{j}, \hat{\beta}_{j}\right)+\left(1-\hat{p}_{j}\right) \cdot f_{\mathcal{N}}\left(Y_{i j} ; X_{i}^{\top} \cdot \hat{\gamma}_{j}, \hat{\sigma}_{j}^{2}\right)},
$$

where $f_{\Gamma}\left(\cdot ; \hat{\alpha}_{j}, \hat{\beta}_{j}\right)$ and $f_{\mathcal{N}}\left(\cdot ; X_{i}^{\top} \hat{\gamma}_{j}, \hat{\sigma}_{j}^{2}\right)$ represent the probability density functions of the estimated Gamma and normal components in the mixture model. Otherwise, if the LRT $p$-value $>0.05$, mbImpute concludes that none of taxon $j$ 's abundances need imputation and sets $d_{1 j}=\cdots=d_{n j}=0$.

Based on the $d_{i j}$ 's, mbImpute defines a set $\Omega$ of (sample, taxon) pairs whose abundances are unlikely missing and thus do not need imputation:

$$
\Omega=\left\{(i, j): d_{i j}<d_{\text {thre }}, i=1, \ldots, n ; j=1, \ldots, m\right\},
$$

and a complement set $\Omega^{c}$ containing other (sample, taxon) pairs whose abundances need imputation:

$$
\Omega^{c}=\left\{(i, j): d_{i j} \geq d_{\text {thre }}, i=1, \ldots, n ; j=1, \ldots, m\right\} .
$$

Although $d_{\text {thre }}=0.5$ is used as the default threshold on $d_{i j}$ 's to decide the abundances that need imputation, mbImpute is fairly robust to this threshold choice because most $d_{i j}$ 's are concentrated around 0 or 1 . We show this phenomenon in Additional file 1: Figure S22, which displays the distribution of all the $d_{i j}$ 's in the data from [14-19]. 
To summarize, mbImpute does not impute all zeros in the taxon count matrix; instead, it first identifies the abundances that are likely missing using a mixture-modelling approach, and it then only imputes these values in the next step.

\section{mblmpute step 2: imputation of the missing taxon abundances}

In step 1, mbImpute identifies a set $\Omega$ of the (sample, taxon) pairs whose abundances do not need imputation. To impute the abundances in $\Omega^{c}$, mbImpute first learns inter-sample and inter-taxon relationships from $\Omega$ by training a predictive model for $Y_{i j}$, the abundance of taxon $j$ in sample $i$. The rationale is that taxon $j$ should have similar abundances in similar samples, and that in every sample, the taxa similar to taxon $j$ should have abundances similar to taxon $j$ 's abundance. In addition, sample covariates are assumed to carry predictive information of taxon abundances. Hence, for interpretability and stability reasons, mbImpute uses a linear model to combine the predictive power of similar taxa, similar samples, and sample covariates:

$$
Y_{i j}=Y_{i .}^{\top} \kappa_{j}+Y_{. j}^{\top} \tau_{i}+X_{i}^{\top} \zeta_{j}+\epsilon_{i j},
$$

where $Y_{i} . \in \mathbb{R}_{>0}^{m}$ denotes the $m$ taxa's abundances in sample $i, Y_{. j} \in \mathbb{R}_{>0}^{n}$ denotes taxon $j$ 's abundances in the $n$ samples, $X_{i} \in \mathbb{R}^{q}$ denotes sample $i$ 's covariates (including the intercept), and $\epsilon_{i j}$ is the error term. The parameters to be estimated include $\kappa_{j} \in \mathbb{R}^{m}, \tau_{i} \in$ $\mathbb{R}^{n}$ and $\zeta_{j} \in \mathbb{R}^{q}, i=1, \ldots, n ; j=1, \ldots, m$. Note that $\kappa_{j}$ represents the $m$ taxa's coefficients (i.e., weights) for predicting taxon $j$, with the $j$ th entry set to zero, so that taxon $j$ would not predict itself; $\tau_{i}$ represents the $n$ samples' coefficients (i.e., weights) for predicting sample $i$, with the $i$ th entry set to zero, so that sample $i$ would not predict itself; $\zeta_{j}$ represents the coefficients of sample covariates for predicting taxon $j$. In the model, the first term $Y_{i}^{\top} \kappa_{j}$ borrows information across taxa, the second term $Y_{. j}^{\top} \tau_{i}$ borrows information across samples, and the third term $X_{i}^{\top} \zeta_{j}$ borrows information from sample covariates. The total number of unknown parameters is $m(m-1)+n(n-1)+m q$, while our data $\mathbf{Y}$ and $\mathbf{X}$ together have $n m+n q$ values only. Given that often $m \gg n$, the parameter estimation problem is high dimensional, as the number of parameters far exceeds the number of data points. mbImpute performs regularized parameter estimation by using the Lasso-type $\ell_{1}$ penalty, which leads to good prediction and simultaneously selects predictors (i.e., similar samples and similar taxa) to ease interpretation. That is, mbImpute estimates the above parameters by minimizing the following loss function:

$$
\begin{aligned}
L\left(\left\{\kappa_{j}, \zeta_{j}\right\}_{j=1}^{m},\left\{\tau_{i}\right\}_{i=1}^{n}\right):= & \sum_{(i, j) \in \Omega}\left[Y_{i j}-\left(Y_{i \cdot}^{\top} \kappa_{j}+Y_{. j}^{\top} \tau_{i}+X_{i .}^{\top} \zeta_{j}\right)\right]^{2} \\
& +\lambda\left(\sum_{j=1}^{m} \sum_{j^{\prime} \neq j}^{m} D_{j j^{\prime}}^{\psi}\left|\kappa_{j j^{\prime}}\right|+\sum_{i=1}^{n} \sum_{i^{\prime} \neq i}^{n}\left|\tau_{i i^{\prime}}\right|\right),
\end{aligned}
$$

where $\lambda, \psi \geq 0$ are tuning parameters chosen by cross-validation, $D_{j j^{\prime}}$ represents the phylogenetic distance between taxa $j$ and $j^{\prime}, \kappa_{j j^{\prime}}$ represents the $j^{\prime}$ th element of $\kappa_{j}$, and $\tau_{i i^{\prime}}$ represents the $i^{\prime}$ th element of $\tau_{i}$. Here $D_{j j^{\prime}}^{\psi}$, i.e., $D_{j j^{\prime}}$ to the power of $\psi$, represents the penalty weight of $\left|\kappa_{j j^{\prime}}\right|$ (in our R package implementation, the mbImpute function can take any distance matrix $D$ as input that reflects the relationship among taxa specified by the user.) The intuition is that if two taxa are closer in the phylogenetic tree, they are more closely related in evolution and tend to have more similar DNA sequences and biological functions $[95,100]$, and thus, we want to borrow more information between them. For 
example, if $D_{j_{1} j_{2}}>D_{j_{1} j_{3}}$, i.e., taxa $j_{1}$ and $j_{2}$ are farther away than taxa $j_{1}$ and $j_{3}$ in the phylogenetic tree, then the estimate of $\kappa_{j_{1} j_{2}}$ is more likely to be shrunk to zero than the estimate of $\kappa_{j_{1} j_{3}}$, and mbImpute would use taxon $j_{3}$ 's abundance more than taxon $j_{2}$ 's to predict taxon $j_{1}$ 's abundance. The tuning parameter $\psi$ is introduced because the distance $D_{j j^{\prime}}$, the number of branches connecting taxa $j$ and $j^{\prime}$, may not be the best penalty weight for the prediction purpose. Choosing $\psi$ by cross-validation is expected to enhance the predication accuracy.

mbImpute performs the estimation using the R package glmnet [74] and obtains the parameter estimates: $\hat{\kappa}_{j} \in \mathbb{R}^{m}, \hat{\tau}_{i} \in \mathbb{R}^{n}$, and $\hat{\zeta}_{j} \in \mathbb{R}^{q}, i=1, \ldots, n ; j=1, \ldots, m$. Finally, for $(i, j) \in \Omega^{c}$, the abundance of taxon $j$ in sample $i$ is imputed as:

$$
\hat{Y}_{i j}=Y_{i}^{\top} \hat{\kappa}_{j}+Y_{. j}^{\top} \hat{i}_{i}+X_{i}^{\top} \hat{\zeta}_{j},
$$

and mbImpute does not alter $Y_{i j}$ if $(i, j) \in \Omega$.

Note that mbImpute does not require the availability of the sample covariate matrix $\mathbf{X}$ or the phylogenetic tree. In the absence of sample covariates, the loss function becomes

$L\left(\left\{\kappa_{j}\right\}_{j=1}^{m},\left\{\tau_{i}\right\}_{i=1}^{n}\right):=\sum_{(i, j) \in \Omega}\left(Y_{i j}-\left(Y_{i \cdot}^{\top} \kappa_{j}+Y_{. j}^{\top} \tau_{i}\right)\right)^{2}+\lambda\left(\sum_{j=1}^{m} \sum_{j^{\prime} \neq j}^{m} D_{j j^{\prime}}^{\psi}\left|\kappa_{j j^{\prime}}\right|+\sum_{i=1}^{n} \sum_{i^{\prime} \neq i}^{n}\left|\tau_{i i^{\prime}}\right|\right)$,

minimizing which returns the parameter estimates: $\hat{\kappa}_{j} \in \mathbb{R}^{m}$ and $\hat{\tau}_{i} \in \mathbb{R}^{n}, i=1, \ldots, n$; $j=1, \ldots, m$. Finally, for $(i, j) \in \Omega^{c}$, the abundance of taxon $j$ in sample $i$ is imputed as:

$$
\hat{Y}_{i j}=Y_{i .}^{\top} \hat{\kappa}_{j}+Y_{. j}^{\top} \hat{\tau}_{i},
$$

and mbImpute does not alter $Y_{i j}$ if $(i, j) \in \Omega$. In the absence of the phylogenetic tree, mbImpute sets $D_{j j^{\prime}}=1$ for all $j \neq j^{\prime} \in\{1, \ldots, m\}$.

When $m$ is large, mbImpute does not estimate $m(m-1)+n(n-1)+m q$ parameters but uses the following strategy to increase its computational efficiency. For each taxon $j$, mbImpute selects the $k$ taxa closest to it (excluding itself) in phylogenetic distance and sets the other $(m-k)$ taxa's coefficients in $\kappa_{j}$ to zero. This strategy reduces the number of parameters to $m k+n(n-1)+m q$ and decreases the computational complexity from $O\left(m^{2}\right)$ to $O(m)$.

In summary, mbImpute step 2 includes two phases: training on $\Omega$ and prediction (imputation) on $\Omega^{c}$, as illustrated in Additional file 1: Figure S1.

\section{Supplementary Information}

The online version contains supplementary material available at https://doi.org/10.1186/s13059-021-02400-4.

Additional file 1: Supplementary materials. It includes simulation settings, analysis details, and supplementary tables and figures.

Additional file 2: DA taxa identified by DESeq2-phyloseq from the Qin et al. T2D dataset [19] with or without imputation. Additional file 3: DA taxa identified by DESeq2-phyloseq from the Karlsson et al. T2D dataset [18] with or without imputation.

Additional file 4: DA taxa identified by DESeq2-phyloseq from the Feng et al. CRC dataset [15] with or without imputation.

Additional file 5: DA taxa identified by DESeq2-phyloseq from the Vogtmann et al. CRC dataset [17] with or without imputation.

Additional file 6: DA taxa identified by DESeq2-phyloseq from the Yu et al. CRC dataset [16] with or without imputation.

Additional file 7: DA taxa identified by DESeq2-phyloseq from the Zeller et al. CRC dataset [14] with or without imputation.

Additional file 8: Review history. 


\section{Acknowledgements}

The authors would like to thank Dr. Hongzhe Li at University of Pennsylvania for pointing us to this research direction. The authors also appreciate the comments and feedback from the members of the Junction of Statistics and Biology at UCLA (http://jsb.ucla.edu).

\section{Peer review information}

Andrew Cosgrove was the primary editor of this article and managed its editorial process and peer review in collaboration with the rest of the editorial team.

\section{Review history}

The review history is available as Additional file 8.

\section{Authors' information}

Twitter handles: @ruochenj1 (Ruochen Jiang); @vivianstats (Wei Vivian Li); @jsb_ucla (Jingyi Jessica Li).

\section{Authors' contributions}

$R J$ and JJL designed the research. RJ conducted the research and wrote the computer code. JJL supervised the project. $R J, W V L$ and JJL discussed the results and wrote the manuscript. The authors read and approved the final manuscript.

\section{Funding}

This work was supported by the following grants: National Science Foundation DMS-1613338 and DBI-1846216, NIH/NIGMS R01GM120507, PhRMA Foundation Research Starter Grant in Informatics, Johnson \& Johnson WiSTEM2D Award, Sloan Research Fellowship, and UCLA David Geffen School of Medicine W.M. Keck Foundation Junior Faculty Award (to J.J.L); Rutgers School of Public Health Pilot Grant and NJ ACTS BERD Mini-Methods Grant (to W.V.L).

\section{Availability of data and materials}

\section{Imputation methods}

We compare mblmpute with five existing imputation methods designed for non-microbiome data: softlmpute and four scRNA-seq imputation methods (scImpute, SAVER, MAGIC, and ALRA). All these imputation methods take a count matrix as input and ouput an imputed count matrix with the same dimensions.

\section{1. softlmpute}

We use R package soft Impute (version 1.4) and the following command to impute an taxon count matrix (a sample-by-taxon matrix):

complete (taxa_count_matrix, softImpute(taxa_count_matrix, rank.max = cv.rankmax))

where rank . max is chosen by 10-fold cross-validation.

\section{2. sclmpute}

We use R package sc Impute (version 0.0.9) with the input as a taxon-by-sample count matrix (transpose of the matrix in Fig. 1):

scimpute (count_path = "taxa_count_matrix_trans.csv", Kcluster = 1, out_dir = "sim imp")

where taxa_count_matrix_trans.csv is the input file containing the transposed taxon count matrix.

\section{SAVER}

We use R package SAVER (version 1.1.2) with the input as a taxon-by-sample count matrix (transpose of the matrix in Fig. 1):

saver(t(taxa_count_matrix), ncores $=1$, estimates.only = TRUE)

\section{MAGIC}

We use Python package MAGIC (version 2.0.3) and the following commands to impute an taxon count matrix: magic_op = magic.MAGIC ()

magic op. set params $(n$ pca $=40)$

magic_op.fit_transform(taxa_count_matrix)

\section{ALRA}

We apply R functions normalize_data, choose_k, and alra, which were released on Aug 10, 2019 at https:// github.com/KlugerLab/ALRA, and the following commands to impute a taxon count matrix: normalized_mat $=$ normalize_data(taxa_count_matrix) $\mathrm{k}$ _chosen $=$ choose_k (normalized_mat, $\overline{\mathrm{K}}=49$, noise_start $=44) \$ \mathrm{k}$ āira (normalized_mät, $\mathrm{k}=\mathrm{k} \_$chosen) $\$$ A_norm_rank_k_cor_sc

\section{DA analysis methods}

In simulation studies, we compare five existing DA methods: the Wilcoxon rank-sum test, ANCOM, metagenomeSeq, DESeq2-phyloseq, and Omnibus test. We apply each method to taxon counts, with or without using mblmpute as a preceding step. When mblmpute is used as a preceding step, we call the resulting method a mblmpute-empowered DA 
method. In real data studies, we compare mblmpute-empowered DESeq2-phyloseq and mblmpute-empowered Omnibus test with DESeq2-phyloseq and Omnibus test, respectively. Each method calculates a $p$ value for each taxon and identifies the DA taxa by setting a $p$ value threshold to control the FDR. See Additional file 1 for the statistical definitions of DA taxa.

\section{Wilcoxon rank-sum test}

We implement the Wilcoxon rank-sum test using the $\mathrm{R}$ function pairwise. wi lcox. test in the package stats (version 3.5.1). For each taxon, we perform the test on its counts in two sample groups to obtain a $p$ value, which suggests if this taxon is DA between the two groups. In simulations, we use the following command to implement a two-sided test for each taxon:

pairwise.wilcox.test ( $\mathrm{x}=$ taxon_counts, $\mathrm{g}=$ condition, $\mathrm{p}$.adjust.method = "none")

\section{ANCOM}

We use the ANCOM . main function released on Sep 27, 2019 at https://github.com/FrederickHuangLin/ANCOM [27]. Since this function does not provide an option for a one-sided test, we use its default settings and report its identified DA taxa based on a two-sided test with a significance level 0.05 ( $\mathrm{ig}=0.05$ ), in both simulations and real data analysis. We note that no external FDR control is implemented. Specifically, we use the following command to obtain the result of ANCOM:

ANCOM.main(taxa count matrix, covariate matrix, adjusted $=F$, repeated $=F$, main.var = "condition", adj.formula = NULL, repeat.var = NULL, multcorr $=2$, sig $=0.05$, prev.cut $=0.90$, longitudinal $=\mathrm{F}$ )

where taxa_count_matrix is a sample-by-taxon count matrix and covariate_matrix is a sample-by-covariate matrix, same $\bar{a}$ the input of mblmpute.

\section{3. metagenomeSeq}

We use two R packages, metagenomeseq (version 1.28.2) and phyloseq (version 1.30.0). Specifically, we use the following command to obtain the result:

mseq_obj <- phyloseq_to_metagenomeseq (physeq2)

pd <- pData (mseq_obj)

mod <- model.matrix $(\sim 1+$ condition, data $=$ pd $)$

ran_seq <- fitFeatureModel (mseq_obj, mod)

where physeq 2 is an object created from a count matrix and sample covariates using the phyloseq package.

\section{DESeq2-phyloseq}

We use the DESeq2 (version 1.26.0) package combined with phyloseq (version 1.30.0). Specifically, we use the following command to obtain the result of DESeq2:

Deseq2_obj <- phyloseq_to_deseq2 (physeq2, condition)

result $\bar{s}<-$ DESeq (Deseq2_obj, test="Wald", fitType="parametric")

where physeq2 is an object created from a count matrix and sample covariates using the phyloseq package.

\section{Omnibus test}

We use the R package mbzinb (version 0.2). Specifically, we use the following command to obtain the result of Omnibus test:

mbzinb data <- mbzinb. dataset (taxa count matrix, covariate matrix)

mbzinb_test_result <- mbzinb.test(mbzinb_data, group = "condition")

For the Wilcoxon rank-sum test, MetagenomeSeq, DESeq2-phyloseq, and Omnibus test, after obtaining the $p$ values of all taxa and collecting them into a vector $p_{-}$values, we adjust them for FDR control using the R function $\mathrm{p}$. adjust in the package stats (version 3.5.1):

p.adjust (p_values, method = "fdr")

Then, we set the FDR threshold to 0.05 in both simulation and real data analysis. The taxa whose adjusted $p$ values do not exceed this threshold are called DA. ANCOM directly outputs the DA taxa.

\section{Classification}

We use a 5-fold cross-validated precision-recall area under the curve (PR-AUC) to evaluate the classification results using identified DA taxa as features and diseased/control group as classification labels. We use the R package randomForest (version 4.6-14) to perform the random forest classification and the R package PRROC (version 1.3.1) to calculate the PR-AUC.

\section{T2D and CRC datasets}

We apply mblmpute to six real microbiome datasets, each corresponding to an independent study on the relationship between microbiomes and the occurrence of a human disease. All the six datasets were generated by the whole genome shotgun sequencing and are available in the R package curatedMetagenomicData [87]. We compare the disease-enriched DA taxa identified by DESeq2-phyloseq and mblmpute-empowered DESeq2-phyloseq. Below is the description of the six datasets and our analysis.

Two T2D datasets $[18,19]$. The Karlsson et al. dataset [18] contains 145 fecal samples from 70-year-old European women to study the relationship between human gut microbiome compositions and T2D status. The samples/subjects 
are in three groups: 53 women with T2D, 49 women with impaired glucose tolerance (IGT), and 43 women as the normal control (CON). The eleven sample covariates include the subject's age, the number of reads in each sample, the triglycerides level, the hba1c level, the Idl (low-density lipoprotein cholesterol) level, the c peptide level, the cholesterol level, the glucose level, the adiponectin level, the hscrp level, and the leptin level. In our analysis, we consider the 147 species-level taxa (having at least 10\% non-zero counts in both T2D and CON groups) with phylogenetic information available in the R package curatedMetagenomicData. Qin et al. [19] performed deep shotgun metagenome sequencing on 369 Chinese T2D patients and non-diabetic controls (CON). The two sample covariates include the body mass index, and the number of reads in each sample. We analyze 156 species-level taxa (having at least 10\% non-zero counts in both T2D and CON groups) with phylogenetic information. From both datasets, we identify DA taxa by comparing the $T 2 \mathrm{D}$ and $\mathrm{CON}$ groups.

Four CRC datasets [14-17]. Zeller et al. [14] and Feng et al. [15] studied CRC-related microbiomes in three conditions: CRC, small adenoma (ADE; diameter $<10 \mathrm{~mm}$ ), and control (CON). Zeller et al. [14] sequenced the fecal samples of patients across two countries (France and Germany) in these three groups: 191 patients with CRC, 66 patients with ADE, and 42 patients in CON. The sample covariates include the subject's age category, gender, body mass index and country, and the number of reads in each sample. We include 188 species-level taxa (having at least 10\% non-zero counts in both CRC and CON groups) with phylogenetic information. Feng et al. [15] sequenced samples from 154 human subjects aged between 45-86 years old in Australia, including 46 patients with CRC, 47 patients with ADE, and 61 in CON. The sample covariates include the subject's age category, gender, body mass index, and number of reads in each sample. We include 182 species-level taxa that have at least 10\% non-zero counts in both CRC and CON groups. Yu et al. [16] and Vogtmann et al. [17] studied CRC-related microbiomes in two conditions: CRC vs. CON. In detail, [16] sequenced 128 Chinese samples, including 75 patients with CRC and 53 patients in CON. The only sample covariate is the number of reads in each sample. We study 173 species-level taxa that have at least 10\% non-zero counts in both CRC and CON groups. Vogtmann et al. [17] included 104 samples from Washington DC and sequenced their fecal samples, including 52 with CRC and 52 in CON. The sample covariates include the subject's age category, gender, body mass index, and the number of reads in each sample. We include 167 species-level taxa that have at least 10\% non-zero counts in both CRC and CON groups. From all the four datasets, we identify DA taxa by comparing the CRC and CON groups.

\section{S rRNA sequencing datasets}

We include two $16 \mathrm{~S}$ rRNA sequencing datasets from the R package HMP16SData [135] (version 1.6.0). The two datasets correspond to the healthy human stool samples and healthy human oral samples. The healthy stool 165 dataset includes 187 samples and 43140 OTUs, and the healthy oral 165 data includes 190 samples and 43140 OTUs.

\section{Software and code}

The mb Impute R package is available at https://github.com/ruochenj/mblmpute [136]. The source code and data for reproducing the results are available at https://doi.org/10.5281/zenodo.4840266 [137]. Both the R package and the source code are under the MIT license.

\section{Declarations}

\section{Ethics approval and consent to participate}

Not applicable.

\section{Competing interests}

The authors declare that they have no competing interests.

\section{Author details}

${ }^{1}$ Department of Statistics, University of California, Los Angeles 90095-1554, CA, USA. ${ }^{2}$ Department of Biostatistics and Epidemiology, Rutgers School of Public Health, Piscataway 08854, NJ, USA. ${ }^{3}$ Department of Human Genetics, University of California, Los Angeles 90095-7088, CA, USA. ${ }^{4}$ Department of Computational Medicine, University of California, Los Angeles 90095-1766, CA, USA. ${ }^{5}$ Department of Biostatistics, University of California, Los Angeles 90095-1772, CA, USA.

Received: 12 February 2021 Accepted: 4 June 2021

Published online: 28 June 2021

\section{References}

1. Katherine RA. An introduction to microbiome analysis for human biology applications. Am J Hum Biol. 2017;29(1): e22931.

2. Turnbaugh PJ, Ley RE, Mahowald MA, Magrini V, Mardis ER, Gordon Jl. An obesity-associated gut microbiome with increased capacity for energy harvest. Nature. 2006;444(7122):1027.

3. Samuel BS, Gordon Jl. A humanized gnotobiotic mouse model of host-archaeal-bacterial mutualism. Proc Natl Acad Sci. 2006;103(26):10011-6

4. Stokholm J, Blaser MJ, Thorsen J, Rasmussen MA, Waage J, Vinding RK, Schoos A-MM, Kunøe A, Fink NR, Chawes BL, et al. Maturation of the gut microbiome and risk of asthma in childhood. Nat Commun. 2018;9(1):1-10.

5. Pragman AA, Kim HB, Reilly CS, Wendt C, Isaacson RE. The lung microbiome in moderate and severe chronic obstructive pulmonary disease. PloS ONE. 2012;7(10):e47305.

6. Holmes E, Li JV, Athanasiou T, Ashrafian H, Nicholson JK. Understanding the role of gut microbiome-host metabolic signal disruption in health and disease. Trends Microbiol. 2011;19(7):349-59.

7. Xinyan Z, Himel M, Nengjun Y. Zero-inflated negative binomial regression for differential abundance testing in microbiome studies. J Bioinforma Genomics. 2016;2(2):. https://doi.org/10.18454/jbg.2016.2.2.1. http://journalbiogen.org/article/view/12. 
8. Besser J, Carleton HA, Gerner-Smidt P, Lindsey RL, Trees E. Next-generation sequencing technologies and their application to the study and control of bacterial infections. Clin Microbiol Infect. 2018;24(4):335-41.

9. Luz Calle M. Statistical analysis of metagenomics data. Genomics Inf. 2019;17(1):e6-.

10. Jovel J, Patterson J, Wang W, Hotte N, O'Keefe S, Mitchel T, Perry T, Kao D, Mason AL, Madsen KL, et al. Characterization of the gut microbiome using 16s or shotgun metagenomics. Front Microbiol. 2016;7:459.

11. Callahan BJ, McMurdie PJ, Rosen MJ, Han AW, Johnson AJA, Holmes SP. DADA2: high-resolution sample inference from Illumina amplicon data. Nat Methods. 2016;13(7):581-3.

12. Callahan BJ, McMurdie PJ, Holmes SP. Exact sequence variants should replace operational taxonomic units in marker-gene data analysis. ISME J. 2017;11(12):2639-43.

13. Li H. Microbiome, Metagenomics, and High-Dimensional Compositional Data Analysis. Annu Rev Stat Appl. 2015;2(1):73-94. https://doi.org/10.1146/annurev-statistics-010814-020351.

14. Zeller G, Tap J, Voigt AY, Sunagawa S, Kultima JR, Costea Pl, Amiot A, Böhm J, Brunetti F, Habermann N, et al. Potential of fecal microbiota for early-stage detection of colorectal cancer. Mol Syst Biol. 2014;10(11):

15. Feng Q, Liang S, Jia H, Stadlmayr A, Tang L, Lan Z, Zhang D, Xia H, Xu X, Jie Z, et al. Gut microbiome development along the colorectal adenoma-carcinoma sequence. Nat Commun. 2015;6:6528.

16. Yu J, Feng Q, Wong SH, Zhang D, Liang Q, Qin Y, Tang L, Zhao H, Stenvang J, Li Y, et al. Metagenomic analysis of faecal microbiome as a tool towards targeted non-invasive biomarkers for colorectal cancer. Gut. 2017;66(1): 70-8. https://pubmed.ncbi.nlm.nih.gov/26408641/.

17. Vogtmann E, Hua X, Zeller G, Sunagawa S, Voigt AY, Hercog R, Goedert JJ, Shi J, Bork P, Sinha R. Colorectal cancer and the human gut microbiome: reproducibility with whole-genome shotgun sequencing. PloS ONE. 2016;11(5):

18. Karlsson FH, Tremaroli V, Nookaew I, Bergström G, Behre CJ, Fagerberg B, Nielsen J, Bäckhed F. Gut metagenome in european women with normal, impaired and diabetic glucose control. Nature. 2013;498(7452):99-103.

19. Qin J, Li Y, Cai Z, Li S, Zhu J, Zhang F, Liang S, Zhang W, Guan Y, Shen D, et al. A metagenome-wide association study of gut microbiota in type 2 diabetes. Nature. 2012;490(7418):55-600.

20. Calgaro M, Romualdi C, Waldron L, Risso D, Vitulo N. Assessment of single cell rna-seq statistical methods on microbiome data. Genome Biology. 2020;21(1):191.

21. Brill B, Amir A, Heller R. Testing for differential abundance in compositional counts data, with application to microbiome studies; 2020. arXiv:1904.08937v5.

22. Silverman JD, Roche K, Mukherjee S, David LA. Naught all zeros in sequence count data are the same. BioRxiv. 2020477794

23. Pereira-Marques J, Anne H, Ferreira RM, Weber M, Pinto-Ribeiro I, van Doorn L-J, Knetsch CW, Figueiredo C. Impact of host DNA and sequencing depth on the taxonomic resolution of whole metagenome sequencing for microbiome analysis. Front Microbiol. 2019;10:1277.

24. Microbiome Human. Project consortium. Structure, function and diversity of the healthy human microbiome Nature. 2012;486:207-14.

25. Lloyd-Price J, Mahurkar A, Rahnavard G, Crabtree J, Orvis J, Hall AB, Brady A, Creasy HH, McCracken C, Giglio MG, et al. Strains, functions and dynamics in the expanded human microbiome project. Nature. 2017;550(7674):61-6.

26. Xia F, Chen J, Fung WK, Li H. A logistic normal multinomial regression model for microbiome compositional data analysis. Biometrics. 2013;69(4):1053-63.

27. Mandal S, Van Treuren W, White RA, Eggesbø M, Knight R, Peddada SD. Analysis of composition of microbiomes: a novel method for studying microbial composition. Microb Ecol Health Dis. 2015;26(1):27663.

28. Tsilimigras MCB, Fodor AA. Compositional data analysis of the microbiome: fundamentals, tools, and challenges. Ann Epidemiol. 2016;26(5):330-35.

29. Weiss S, Xu ZZ, Peddada S, Amir A, Bittinger K, Gonzalez A, Lozupone C, Zaneveld JR, Vázquez-Baeza Y, Birmingham A, et al. Normalization and microbial differential abundance strategies depend upon data characteristics. Microbiome. 2017;5(1):27.

30. Kaul A, Mandal S, Davidov O, Peddada SD. Analysis of microbiome data in the presence of excess zeros. Front Microbiol. 2017:8:2114.

31. Lizhen X, Paterson AD, Turpin W, Wei X. Assessment and selection of competing models for zero-inflated microbiome data. PloS ONE. 2015;10(7):

32. Chen J, King E, Deek R, Wei Z, Yue Y, Grill D, Ballman K. An omnibus test for differential distribution analysis of microbiome sequencing data. Bioinformatics. 2018a;34(4):643-51.

33. McMurdie PJ, Holmes S. phyloseq: an r package for reproducible interactive analysis and graphics of microbiome census data. PloS ONE. 2013;8(4):e61217.

34. Love MI, Huber W, Anders S. Moderated estimation of fold change and dispersion for RNA-seq data with DESeq2. Genome Biol. 2014;15(12):550.

35. Paulson JN, Colin Stine O. Héctor Corrada Bravo, and Mihai Pop. Differential abundance analysis for microbial marker-gene surveys. Nat Methods. 2013;10(12):1200-2.

36. Peng X, Li G, Liu Z. Zero-inflated beta regression for differential abundance analysis with metagenomics data. J Comput Biol. 2016;23(2):102-10.

37. Randolph TW, Zhao S, Copeland W, Hullar M, Shojaie A. Kernel-penalized regression for analysis of microbiome data. Ann Appl Stat. 2018;12(1):540.

38. Li Z, Lee K, Karagas MR, Madan JC, Hoen AG, O'malley AJ, Li H. Conditional regression based on a multivariate zero-inflated logistic-normal model for microbiome relative abundance data. Stat Biosci. 2018;10(3):587-608.

39. Hawinkel Stijn, Mattiello Federico, Bijnens Luc, Thas Olivier. A broken promise: microbiome differential abundance methods do not control the false discovery rate. Brief Bioinforma. 2019;20(1):210-21.

40. Horner-Devine MC, Silver JM, Leibold MA, Bohannan BJM, Colwell RK, Fuhrman JA, Green JL, Kuske CR, Martiny $\mathrm{JBH}$, Muyzer $\mathrm{G}$, et al. A comparison of taxon co-occurrence patterns for macro-and microorganisms. Ecology. 2007;88(6):1345-53.

41. Barberán A, Bates ST, Casamayor EO, Fierer N. Using network analysis to explore co-occurrence patterns in soil microbial communities. ISME J. 2012;6(2):343-51.

42. Gokul JK, Hodson AJ, Saetnan ER, Irvine-Fynn TDL, Westall PJ, Detheridge AP, Takeuchi N, Bussell J, Mur LAJ, Edwards A. Taxon interactions control the distributions of cryoconite bacteria colonizing a high arctic ice cap. Mol Ecol. 2016;25(15):3752-67. 
43. Tapio I, Fischer D, Blasco L, Tapio M, Wallace RJ, Bayat AR, Ventto L, Kahala M, Negussie E, Shingfield KJ, et al. Taxon abundance, diversity, co-occurrence and network analysis of the ruminal microbiota in response to dietary changes in dairy cows. PloS ONE. 2017;12(7)::

44. Bennett J, Lanning S, et al. The Netflix prize. In: Proceedings of KDD cup and workshop, vol 2007. Citeseer; 2007. p. 35.

45. Dass SC, Nair VN. Edge detection, spatial smoothing, and image reconstruction with partially observed multivariate data. J Am Stat Assoc. 2003;98(461):77-89.

46. Faubel F, McDonough J, Dietrich K. Bounded conditional mean imputation with Gaussian mixture models: a reconstruction approach to partly occluded features. In: 2009 IEEE International Conference on Acoustics, Speech and Signal Processing. IEEE; 2009. p. 3869-72.

47. Rulloni V, Bustos O, Flesia AG. Large gap imputation in remote sensed imagery of the environment. Comput Stat Data Anal. 2012;56(8):2388-2403.

48. Ernst Jason, Kellis Manolis. Large-scale imputation of epigenomic datasets for systematic annotation of diverse human tissues. Nat Biotechnol. 2015;33(4):364.

49. Marchini Jonathan, Howie Bryan. Genotype imputation for genome-wide association studies. Nat Rev Genet. 2010;11(7):499-511.

50. Li WW, Li JJ. An accurate and robust imputation method scimpute for single-cell RNA-seq data. Nat Commun 2018;9(1):1-9.

51. Van Dijk D, Sharma R, Nainys J, Yim K, Kathail P, Carr AJ, Burdziak C, Moon KR, Chaffer CL, Pattabiraman D, et al. Recovering gene interactions from single-cell data using data diffusion. Cell. 2018;174(3):716-29.

52. Mo H, Wang J, Torre E, Dueck H, Shaffer S, Bonasio R, Murray Jl, Raj A, Li M, Zhang NR. SAVER: gene expression recovery for single-cell RNA sequencing. Nat Methods. 2018;15(7):539-42.

53. Linderman GC, Zhao J, Kluger Y. Zero-preserving imputation of scRNA-seq data using low-rank approximation. bioRxiv. 2018397588.

54. Eraslan G, Simon LM, Mircea M, Mueller NS, Theis FJ. Single-cell RNA-seq denoising using a deep count autoencoder. Nat Commun. 2019;10(1):390.

55. Martino C, Morton JT, Marotz CA, Thompson LR, Tripathi A, Knight R, Zengler K. A novel sparse compositional technique reveals microbial perturbations. MSystems. 2019;4(1):

56. Cai Yun, Hong Gu, Kenney Toby. Learning microbial community structures with supervised and unsupervised non-negative matrix factorization. Microbiome. 2017;5(1):110.

57. Garamszegi LZ. Modern phylogenetic comparative methods and their application in evolutionary biology: concepts and practice: Springer; 2014

58. Revell LJ. phytools: an R package for phylogenetic comparative biology (and other things). Methods Ecol Evol. 2012;3(2):217-23.

59. Kembel SW, Cowan PD, Helmus MR, Cornwell WK, Morlon H, Ackerly DD, Blomberg SP, Webb CO. Picante: R tools for integrating phylogenies and ecology. Bioinformatics. 2010;26(11):1463-64.

60. Orme D, Freckleton R, Thomas G, Petzoldt T, Fritz S, Isaac N, et al. The caper package: comparative analysis of phylogenetics and evolution in R. R package version. 2013;5(2):1-36.

61. Gloor GB, Reid G. Compositional analysis: a valid approach to analyze microbiome high-throughput sequencing data. Can J Microbiol. 2016;62(8):692-703.

62. Chen J, Bushman FD, Lewis JD, Wu GD, Li H. Structure-constrained sparse canonical correlation analysis with an application to microbiome data analysis. Biostatistics. 2013;14(2):244-58.

63. Wang T, Zhao H. Constructing predictive microbial signatures at multiple taxonomic levels. J Am Stat Assoc. 2017;112(519):1022-31.

64. Xiao J, Cao H, Chen J. False discovery rate control incorporating phylogenetic tree increases detection power in microbiome-wide multiple testing. Bioinformatics. 2017;33(18):2873-81.

65. Washburne AD, Morton JT, Sanders J, McDonald D, Zhu Q, Oliverio AM, Knight R. Methods for phylogenetic analysis of microbiome data. Nat Microbiol. 2018;3(6):652-61.

66. Anderson TM, Lachance M-A, Starmer WT. The relationship of phylogeny to community structure: the cactus yeast community. Am Nat. 2004:164(6):709-21.

67. Webb CO, Ackerly DD, McPeek MA, Donoghue MJ. Phylogenies and community ecology. Annu Rev Ecol Syst. 2002;33(1):475-505.

68. Weiher E, Keddy PA. Assembly rules, null models, and trait dispersion: new questions from old patterns. Oikos. 1995159-64.

69. Arisdakessian C, Poirion O, Yunits B, Zhu X, Garmire LX. Deepimpute: an accurate, fast, and scalable deep neural network method to impute single-cell RNA-seq data. Genome Biol. 2019;20(1):1-14.

70. Hastie T, Mazumder R. softimpute: Matrix completion via iterative soft-thresholded svd. R package version. 2015;p1:1.

71. Allen-Vercoe E, Jobin C. Fusobacterium and Enterobacteriaceae: important players for CRC Immunol Lett. 2014;162(2):54-61.

72. Dandona P, Aljada A, Bandyopadhyay A. Inflammation: the link between insulin resistance, obesity and diabetes. Trends Immunol. 2004;25(1):4-7.

73. Dulal S, Keku TO. Gut microbiome and colorectal adenomas. Cancer J (Sudbury, Mass.) 2014;20(3):225.

74. Friedman J, Hastie T, Tibshirani R. glmnet: Lasso and elastic-net regularized generalized linear models. R package version. 2009;1(4):.

75. Gong W, Kwak I-Y, Pota P, Koyano-Nakagawa N, Garry DJ. Drimpute: imputing dropout events in single cell RNA sequencing data. BMC Bioinformatics. 2018;19(1):220.

76. Jackman S. pscl: classes and methods for R. Developed in the Political Science Computational Laboratory, Stanford University. Department of Political Science, Stanford University, Stanford, CA. r package version 1.03.5. 2010. http:// www.pscl.stanford.edu/. Accessed 15 Dec 2020

77. Kalisch M, Bühlman P. Estimating high-dimensional directed acyclic graphs with the PC-algorithm. J Mach Learn Res. 2007;8(3):613-36. 
78. Krijthe JH. Rtsne: T-distributed stochastic neighbor embedding using Barnes-Hut implementation. R package version 0.13. 2015. https://github.com/jkriithe/Rtsne. Accessed 15 Dec 2020.

79. Larsen N, Vogensen FK, Van Den Berg FWJ, Nielsen DS, Andreasen AS, Pedersen BK, Al-Soud WA, Sørensen SJ, Hansen LH, Jakobsen M. Gut microbiota in human adults with type 2 diabetes differs from non-diabetic adults. PloS ONE. 2010;5(2):

80. Ley RE, Bäckhed F, Turnbaugh P, Lozupone CA, Knight RD, Gordon Jl. Obesity alters gut microbial ecology. Proc Natl Acad Sci. 2005;102(31):11070-5.

81. Li WV, Li JJ. A statistical simulator scDesign for rational scRNA-seq experimental design. Bioinformatics. 2019;35(14): i41-i50.

82. Martínez I, Lattimer JM, Hubach KL, Case JA, Yang J, Weber CG, Louk JA, Rose DJ, Kyureghian G, Peterson DA, et al. Gut microbiome composition is linked to whole grain-induced immunological improvements. ISME J. 2013;7(2): 269-80.

83. Nakatsu G, Li X, Zhou H, Sheng J, Wong SH, Wu WKK, Ng SC, Tsoi H, Dong Y, Zhang N, et al. Gut mucosal microbiome across stages of colorectal carcinogenesis. Nat Commun. 2015;6(1):1-9.

84. Nguyen NT, Nguyen X-MT, Lane J, Wang P. Relationship between obesity and diabetes in a US adult population: findings from the National Health and Nutrition Examination Survey, 1999-2006. Obes Surg. 2011;21(3):351-55.

85. van Nimwegen KJM, van Soest RA, Veltman JA, Nelen MR, van der Wilt GJ, Vissers LELM, Grutters JPC. Is the 1000 genome as near as we think? A cost analysis of next-generation sequencing. Clin Chem. 2016;62(11):1458-64.

86. Noble D, Mathur R, Dent T, Meads C, Greenhalgh T. Risk models and scores for type 2 diabetes: systematic review. Bmj. 2011;d7163:343.

87. Pasolli E, Schiffer L, Manghi P, Renson A, Obenchain V, Truong DT, Beghini F, Malik F, Ramos M, Dowd JB, et al. Accessible, curated metagenomic data through ExperimentHub. Nat Methods. 2017;14(11):1023.

88. Remely M, Dworzak S, Hippe B, Zwielehner J, Aumüller E, Brath $\mathrm{H}$, Haslberger A. Abundance and diversity of microbiota in type 2 diabetes and obesity. J Diabete Metab. 2013;4(253):2.

89. Ren B, Schwager E, Tickle TL, Huttenhower C. sparseDOSSA: Sparse Data Observations for Simulating Synthetic Abundance. 2016. R package version 0.99.6.

90. Sanapareddy N, Legge RM, Jovov B, McCoy A, Burcal L, Araujo-Perez F, Randall TA, Galanko J, Benson A, Sandler RS, et al. Increased rectal microbial richness is associated with the presence of colorectal adenomas in humans. ISME J. 2012;6(10):1858-68.

91. Savage DC. Microbial ecology of the gastrointestinal tract. Annu Rev Microbiol. 1977;31(1):107-33.

92. Semova I, Carten JD, Stombaugh J, Mackey LC, Knight R, Farber SA, Rawls JF. Microbiota regulate intestinal absorption and metabolism of fatty acids in the zebrafish. Cell Host Microbe. 2012;12(3):277-88.

93. Shen XJ, Rawls JF, Randall TA, Burcall L, Mpande C, Jenkins N, Jovov B, Abdo Z, Sandler RS, Keku TO. Molecular characterization of mucosal adherent bacteria and associations with colorectal adenomas. Gut Microbes. 2010;1(3): $138-47$.

94. Sobhani I, Tap Julien, Roudot-Thoraval F, Roperch JP, Letulle S, Langella P, Corthier G, Van Nhieu JT, Furet JP. Microbial dysbiosis in colorectal cancer (CRC) patients. PloS ONE. 2011;1(6):1-7.

95. Waese J, Provart NJ, Guttman DS. Topo-phylogeny: visualizing evolutionary relationships on a topographic landscape. PloS ONE. 2017;1(5):e0175895.

96. Wang T, Cai G, Qiu Y, Na F, Zhang M, Pang X, Jia W, Cai S, Zhao L. Structural segregation of gut microbiota between colorectal cancer patients and healthy volunteers. ISME J. 2012;6(2):320-9.

97. Weir TL, Manter DK, Sheflin AM, Barnett BA, Heuberger AL, Ryan EP. Stool microbiome and metabolome differences between colorectal cancer patients and healthy adults. PloS ONE. 2013;8(8):1-10.

98. Na Wu, Yang X, Zhang R, Li J, Xiao X, Hu Y, Chen Y, Yang F, Lu N, Wang Z, et al. Dysbiosis signature of fecal microbiota in colorectal cancer patients. Microb Ecol. 2013;66(2):462-70.

99. Sicheng Wu, Sun C, Li Y, Wang T, Jia L, Lai S, Yang Y, Luo P, Dai D, Yang Y-Q, et al. GMrepo: a database of curated and consistently annotated human gut metagenomes. Nucleic Acids Res. 2020;48(D1):D545—D553.

100. Xiao J, Li C, Johnson S, Yue Y, Zhang X, Chen J. Predictive modeling of microbiome data using a phylogeny-regularized generalized linear mixed model. Front Microbiol. 2018;9:1391.

101. Yang Y, Cai Q, Zheng W, Steinwandel M, Blot WJ, Shu X-O, Long J. Oral microbiome and obesity in a large study of low-income and African-American populations. J Oral Microbiol. 2019;11(1):1650597.

102. Chen J, King E, Deek R, Wei Z, Yue Y, Grill D, Ballman K. An omnibus test for differential distribution analysis of microbiome sequencing data. Bioinformatics. 2018b;34(4):643-51.

103. Yanyun Gu, Wang X, Li J, Zhang Y, Zhong H, Liu R, Zhang D, Feng Q, Xie X, Hong J, et al. Analyses of gut microbiota and plasma bile acids enable stratification of patients for antidiabetic treatment. Nat Commun. 2017:8(1):1-12.

104. Horie M, Miura T, Hirakata S, Hosoyama A, Sugino S, Umeno A, Murotomi K, Yoshida Y, Koike T. Comparative analysis of the intestinal flora in type 2 diabetes and nondiabetic mice. Exp Anim. 2017;66:17-0021.

105. Moore WE, Moore LH. Intestinal floras of populations that have a high risk of colon cancer. Appl Environ Microbiol. 1995;61(9):3202-7.

106. Lucas C, Barnich N, Nguyen HTT. Microbiota, inflammation and colorectal cancer. Int J Mol Sci. 2017;18(6):1310.

107. Peterson CT, Sharma V, Elmén L, Peterson SN. Immune homeostasis, dysbiosis and therapeutic modulation of the gut microbiota. Clin Exp Immunol. 2015;179(3):363-77.

108. Shaoguang Wu, Rhee K-J, Albesiano E, Rabizadeh S, Wu X, Yen H-R, Huso DL, Brancati FL, WickE, McAllister F, et al. A human colonic commensal promotes colon tumorigenesis via activation of $\mathrm{T}$ helper type $17 \mathrm{~T}$ cell responses. Nat Mater. 2009;15(9):1016-22.

109. Wang J, Jia H. Metagenome-wide association studies: fine-mining the microbiome. Nat Rev Microbiol. 2016;14(8): 508-22.

110. He G, Wen J-J, Jie-Lun H, Nie Q-X, Chen H-H, Xiong T, Nie S-P, Xie M-Y. Polysaccharide from fermented Momordica charantia L. with Lactobacillus plantarum NCU1 16 ameliorates type 2 diabetes in rats. Carbohydr Polym. 2018;201:624-33. 
111. Kosumi K, Hamada T, Koh H, Borowsky J, Bullman S, Twombly TS, Nevo D, Masugi Y, Liu L, da Silva A, et al. The amount of Bifidobacterium genus in colorectal carcinoma tissue in relation to tumor characteristics and clinical outcome. Am J Pathol. 2018;188(12):2839-52.

112. Parisa A, Roya G, Mahdi R, Shabnam R, Maryam E, Malihe T. Anti-cancer effects of Bifidobacterium species in colon cancer cells and a mouse model of carcinogenesis. PloS ONE. 2020;15(5):e0232930.

113. Bahmani S, Azarpira N, Moazamian E. Anti-colon cancer activity of Bifidobacterium metabolites on colon cancer cell line SW742. Turk J Gastroenterol. 2019;30(9):835.

114. Wang Q, Wang K, Wu W, Lv L, Bian X, Yang L, Wang Q, Li Y, Ye J, Fang D, et al. Administration of Bifidobacterium bifidum CGMCC 15068 modulates gut microbiota and metabolome in azoxymethane (AOM)/dextran sulphate sodium (DSS)-induced colitis-associated colon cancer (CAC) in mice. Appl Microbiol Biotechnol. 2020;104(13):5915-28.

115. Gueimonde M, Ouwehand A, Huhtinen H, Salminen E, Salminen S. Qualitative and quantitative analyses of the bifidobacterial microbiota in the colonic mucosa of patients with colorectal cancer, diverticulitis and inflammatory bowel disease. World J Gastroenterol WJG. 2007;13(29):3985.

116. Fahmy CA, Gamal-Eldeen AM, El-Hussieny EA, Raafat BM, Mehanna NS, Talaat RM, Shaaban MT. Bifidobacterium longum suppresses murine colorectal cancer through the modulation of oncomirs and tumor suppressor mirnas. Nutr Cancer. 2019;71(4):688-700.

117. Chen EZ, Li H. A two-part mixed-effects model for analyzing longitudinal microbiome compositional data. Bioinformatics. 2016;32(17):2611-7.

118. Layeghifard M, Hwang DM, Guttman DS. Disentangling interactions in the microbiome: a network perspective. Trends Microbiol. 2017;25(3):217-28.

119. Turnbaugh PJ, Ley RE, Hamady M, Fraser-Liggett CM, Knight R, Gordon J. The human microbiome project. Nature. 2007;449(7164):804-10.

120. Sugino KY, Paneth N, Comstock SS. Michigan cohorts to determine associations of maternal pre-pregnancy body mass index with pregnancy and infant gastrointestinal microbial communities: late pregnancy and early infancy. PloS ONE. 2019;14(3):e0213733.

121. Yang Q, Lin SL, Kwok MK, Leung GM, Schooling CM. The roles of 27 genera of human gut microbiota in ischemic heart disease, type 2 diabetes mellitus, and their risk factors: a Mendelian randomization study. Am J Epidemiol. 2018;187(9):1916-22.

122. David LA, Maurice CF, Carmody RN, Gootenberg DB, Button JE, Wolfe BE, Ling AV, Devlin AS, Varma Y, Fischbach MA, et al. Diet rapidly and reproducibly alters the human gut microbiome. Nature. 2014;505(7484):559-63.

123. Boulch ML, Déhais $P$, Combes $S$, Pascal G. The MACADAM database: a metabolic pathways database for microbial taxonomic groups for mining potential metabolic capacities of archaeal and bacterial taxonomic groups. Database. 2019;2019:2019.

124. McKnight DT, Huerlimann R, Bower DS, Schwarzkopf L, Alford RA, Zenger KR. Methods for normalizing microbiome data: an ecological perspective. Methods Ecol Evol. 2019;10(3):389-400.

125. Salter SJ, Cox MJ, Turek EM, Calus ST, Cookson WO, Moffatt MF, Turner P, Parkhill J, Loman NJ, Walker AW. Reagent and laboratory contamination can critically impact sequence-based microbiome analyses. BMC Biol. 2014;12(1):1-12.

126. Glassing A, Dowd SE, Galandiuk S, Davis B, Chiodini RJ. Inherent bacterial dna contamination of extraction and sequencing reagents may affect interpretation of microbiota in low bacterial biomass samples. Gut Pathog. 2016;8(1):1-12.

127. Jervis-Bardy J, Leong LEX, Marri S, Smith RJ, Choo JM Smith-Vaughan, Nosworthy E, Morris PS, O'Leary S, Rogers $G B$, et al. Deriving accurate microbiota profiles from human samples with low bacterial content through post-sequencing processing of Illumina MiSeq data. Microbiome. 2015;3(1):1-11.

128. Kirstahler P, Bjerrum SS, Friis-Møller A, Cour ML, Aarestrup FM, Westh H, Pamp SJ. Genomics-based identification of microorganisms in human ocular body fluid. Sci Rep. 2018;8(1):1-14.

129. Karstens L, Asquith M, Davin S, Fair D, Thomas GW, Wolfe AJ, Braun J, McWeeney S. Controlling for contaminants in low-biomass 165 rRNA gene sequencing experiments. MSystems. 2019;4(4):e00290-19.

130. Davis NM, Proctor DM, Holmes SP, Relman DA, Callahan BJ. Simple statistical identification and removal of contaminant sequences in marker-gene and metagenomics data. Microbiome. 2018;6(1):1-14.

131. Efron B, Hastie T. Computer age statistical inference, vol 5: Cambridge University Press; 2016.

132. Poudel R, Jumpponen A, Schlatter DC, Paulitz TC, McSpadden Gardener BB, Kinkel LL, Garrett KA. Microbiome networks: a systems framework for identifying candidate microbial assemblages for disease management. Phytopathology. 2016;106(10):1083-96.

133. Li C, Reeve J, Zhang L, Huang S, Wang X, Gmpr JC. A robust normalization method for zero-inflated count data with application to microbiome sequencing data. PeerJ. 2018c;e4600:6.

134. Manor O, Borenstein E. MUSiCC: a marker genes based framework for metagenomic normalization and accurate profiling of gene abundances in the microbiome. Genome Biol. 2015;16(1):53.

135. Schiffer L, Azhar R, Shepherd L, Ramos M, Geistlinger L, Huttenhower C, Dowd JB, Segata N, Waldron L. HMP16SData: efficient access to the human microbiome project through bioconductor. Am J Epidemiol. 2019. https://doi.org/10.1093/aje/kwz006.

136. Jiang R. mblmpute: an accurate and robust imputation method for microbiome data. URL https://github.com/ ruochenj/mblmpute. R package version 0.1.0. Accessed 15 Dec 2020.

137. Jiang R, Li WV, Li JJ. mblmpute: an accurate and robust imputation method for microbiome data. 2021. URL https://doi.org/10.5281/zenodo.4840266.

\section{Publisher's Note}

Springer Nature remains neutral with regard to jurisdictional claims in published maps and institutional affiliations. 